\title{
New advances in MR-compatible bioartificial liver
}

\section{Rex E. Jeffries ${ }^{a}$ and Jeffrey M. Macdonald ${ }^{a *}$}

\begin{abstract}
MR-compatible bioartificial liver (BAL) studies have been performed for $\mathbf{3 0}$ years and are reviewed. There are two types of study: (i) metabolism and drug studies using multinuclear MRS; primarily short-term ( $<8$ h) studies; (ii) the use of multinuclear MRS and MRI to noninvasively define the features and functions of BAL systems for long-term liver tissue engineering. In the latter, these systems often undergo not only modification of the perfusion system, but also the construction of MR radiofrequency probes around the bioreactor. We present novel MR-compatible BALs and the use of multinuclear MRS $\left({ }^{13} \mathrm{C},{ }^{19} \mathrm{~F},{ }^{31} \mathrm{P}\right)$ for the noninvasive monitoring of their growth, metabolism and viability, as well as ${ }^{1} \mathrm{H}$ MRI methods for the determination of flow profiles, diffusion, cell distribution, quality assurance and bioreactor integrity. Finally, a simple flexible coil design and circuit, and life support system, are described that can make almost any BAL MR-compatible. Copyright (C) 2011 John Wiley \& Sons, Ltd.
\end{abstract}

Keywords: MR-compatible bioreactor; radiofrequency probe construction; bioartificial liver; ${ }^{31} \mathrm{P}$ MRS; ${ }^{13} \mathrm{C}$ MRS; ${ }^{19} \mathrm{~F}$ MRS; ${ }^{1} \mathrm{H}$ MRI

\section{INTRODUCTION}

MRS studies of cell preparations permit the control of cell composition and the evaluation of contributions from immune, endocrine, paracrine and physicochemical parameters not afforded in intact or perfused organ systems [for reviews, see refs. (1-6)]. Since 1973, four categories of MR-compatible mammalian cell perfusion systems have evolved (7): (i) suspension (8); (ii) entrapment (9-12); (iii) microcarrier $(4,13)$; and (iv) membrane (14). Table 1 contains some MR and physiological metrics for comparison of these various bioreactor categories.

Although MR-compatible bioartificial livers (BALs) have been used for three decades (15), the lack of long-term cell viability and the complexity of bioreactor operation have hindered their widespread application in the pharmaceutical industry. Initially, MR-compatible bioreactors for hepatocytes, or BALs, used hepatocyte suspensions, but function and viability only persisted for several hours before hypoxia caused cell death $(8,16-18)$. This is because suspension bioreactors are the only category in Table 1 without perfusion, and therefore mass transfer is the most limited of all categories. However, from an MRS standpoint, they are easy to operate, have excellent global density (i.e. the percentage of sensitive volume in the coil filled by cells) leading to a superior signal-to-noise ratio (SNR), and can be packed in a $5-\mathrm{mm}$ NMR tube, so that standard high-resolution NMR spectrometers can be used.

The goal of these early studies was not to maintain long-term viability, but to obtain short-term metabolic information, and a few hours were all that was required for this goal. This simple suspension BAL evolved to perfusion systems in which hepatocytes were entrapped in collagen threads (19-25) or alginate spherical encapsulates (26-28), or inoculated into membrane bioreactors (29). The most widely applied method for metabolism studies is the entrapment technique, whereby cells are immobilized in threads of agarose $(30)$ or alginate $(9,11)$. All early BAL studies used nonphysiological concentrations of oxygen, typically $95: 5$ oxygen : carbon dioxide (carbogen) (15), in order to overcome hypoxia, whereby, in vivo, the blood contains hemoglobin to reduce the oxygen gradient across the liver (Table 1). In addition, simple salt solutions or nonbasal medium solutions were used (Table 1), which is not permissive for long-term cultures, and more typical of toxicology studies (31). More recently, with the advent of tissue engineering, MRS and, especially, MRI have been used as tools to characterize the bioreactor, with MRS focusing on ${ }^{31} \mathrm{P}$ and viability $(16-26,29)$, and no reported studies of ${ }^{13} \mathrm{C},{ }^{15} \mathrm{~N},{ }^{1} \mathrm{H}$ or ${ }^{19} \mathrm{~F}$ to characterize tissue-engineered BAL function. Recently, dynamic nuclear polarization ${ }^{13} \mathrm{C}$ MRS has been used to determine flux rates in alginate-entrapped rat hepatoma cells perfused in a fluidizedbed BAL (32); however, this review focuses on in vivo MR studies of primary hepatocytes. ${ }^{1} \mathrm{H}$ MRI has been used to investigate inoculation efficiency and distribution, as well as to obtain quality assurance data (29), and velocity-encoded MRI has been used to define the flow characteristics (33); diffusion-weighted MRI and other forms have not yet been applied to BAL. One reason for this lack of MR research is that conventional BAL systems do not fit in vertical bore magnets and require horizontal bore magnets, which typically do not possess a second broad-band channel for multinuclear MRS analysis, and/or there are no easy instructions to make BALs MRS- and MRI-compatible. This review provides an

\footnotetext{
* Correspondence to: J. M. Macdonald, Department of Biomedical Engineering, University of North Carolina, Chapel Hill, NC 27599-7575, USA.

a R. E. Jeffries, J. M. Macdonald

Department of Biomedical Engineering, University of North Carolina at Chapel Hill, Chapel Hill, NC, USA
}

Abbreviations used: ATP, adenosine triphosphate; BAL, bioartificial liver;
ECC, extracapillary compartment; FEPT, fluorinated ethylenepropylene; FIDAP,
Fluid Dynamics Analysis Package; ICC, intracapillary compartment; NTP,
nucleotide triphosphate; Pi(ext), extracellular inorganic phosphate; Pi(int),
intracellular inorganic phosphate; $Q_{\text {rad, }}$ radial flow; SNR, signal-to-noise ratio. 


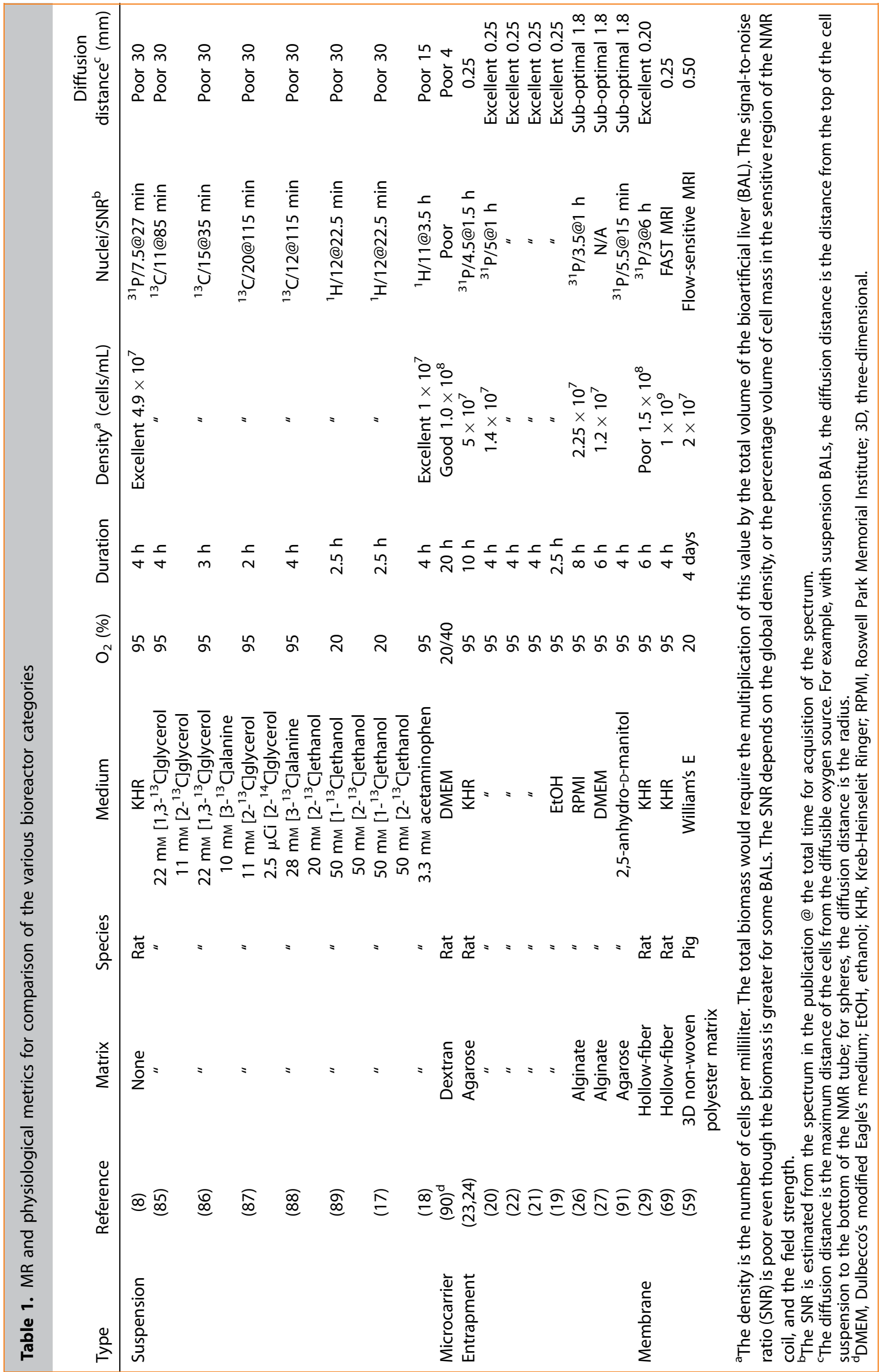


easy step-by-step approach to make any BAL MR-compatible, and presents examples of the application of multinuclear MRS protocols to monitor the viability and function of several BAL designs, as well as MRI applications to characterize inoculation efficiency and distribution in opaque materials, quality assurance, flow dynamics and oxygen distribution.

As suspension BALs were the first design attempted, and are not feasible for long-term maintenance of liver cells in tissue engineering (Table 1), they are not used for the demonstration of MRS and MRI applications in this review. Rather, three bioreactors corresponding to membrane, micro- carrier and entrapment, which have been tested by MRS and MRI in the author's laboratory, are used to demonstrate the applications of MRS and MRI, whilst demonstrating the effects of various bioreactor parameters on hepatocyte viability, such as the diffusion distance, shear forces, channeling and the gelling or aggregation of hepatocytes. Figure 1 includes diagrams and photographs of the three bioreactors that are presented: (i) hollow fiber membrane bioreactor inoculated with microcarriers; (ii) membrane multicoaxial hollow fiber bioreactor; and (iii) fluidized bioreactor consisting of alginateentrapped hepatocytes.
(A)

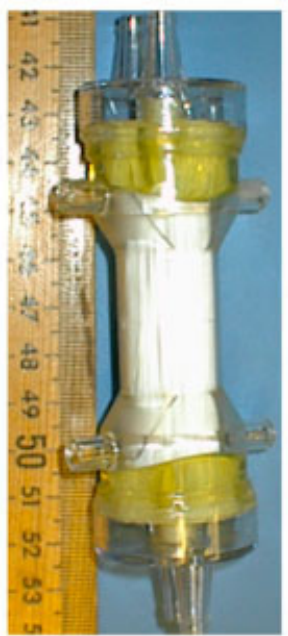

(B)

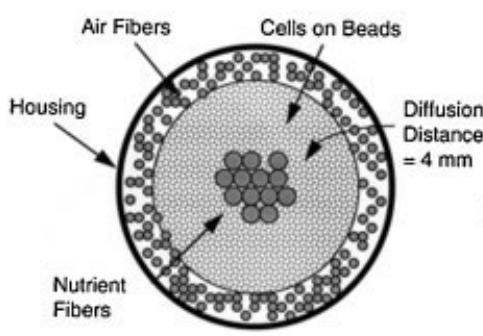

(C)

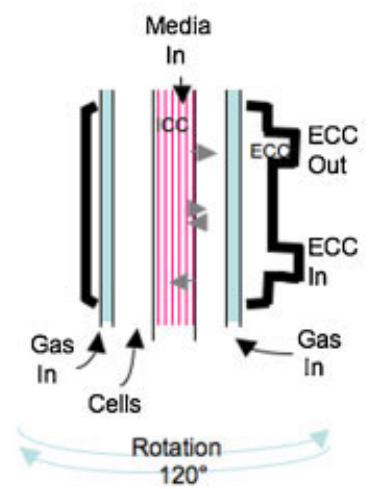

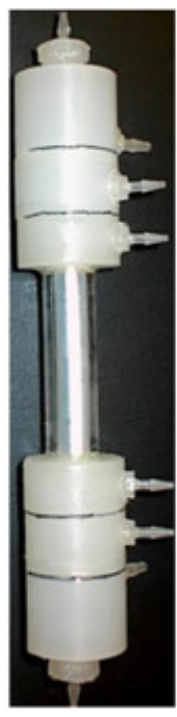

Multicoaxial Membrane
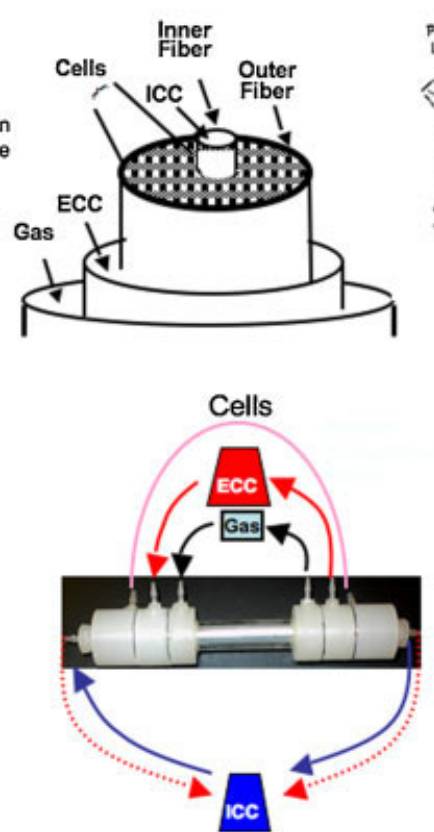

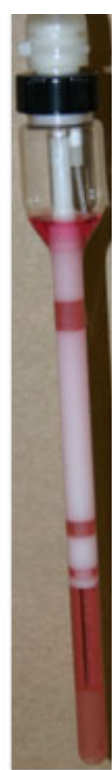

Fluidized-Bed

Encapsulation
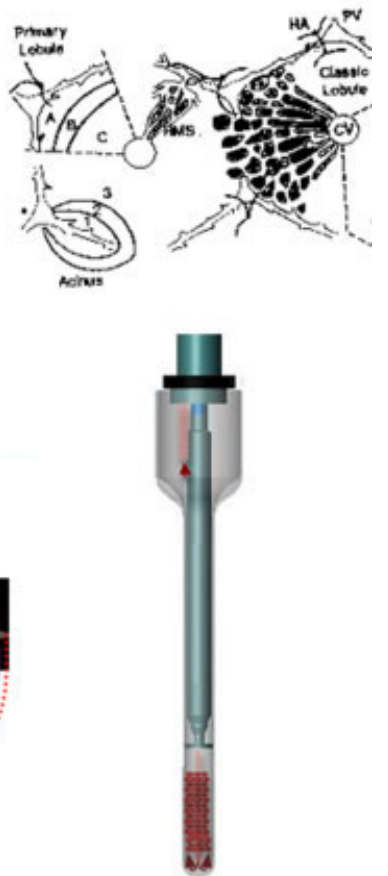

Figure 1. The three bioartificial liver (BAL) designs described: hybrid hollow fiber-microcarrier bioreactor; multicoaxial hollow fiber membrane bioreactor; and fluidized-bed encapsulation bioreactor. Three photographs of the bioreactors (A) with details of their design (B) and flow patterns (C). ECC, extracapillary compartment; ICC, intracapillary compartment. 


\section{MRS AND MRI COMPATIBILITY - BUILDING COMPACT RADIOFREQUENCY (RF) MR PROBES FOR BALS}

MR probes cost tens of thousands of dollars if purchased from a commercial vendor, but can be easily constructed with minimal experience beyond a novice education in RF engineering (34). For animal studies, a knowledge of building one's own MR probe is essential as one may need to change the coil geometries based on the region of interest and, for some BAL designs, this may also be the case. The majority of available NMR spectrometers are vertical bore instruments, and a volume coil is used, wherein the entire central portion of the BAL is contained within the MR coil. For animals, typically surface coils are used to localize to the tissue of interest, and typically the MR probe is designed for a horizontal bore magnet for rats or larger animals. MR probe designs for horizontal and vertical bore magnets are similar, but MR probes for horizontal-bore magnets have larger cradles and typically do not need the RF aluminum casing to shield the electronics from the room temperature shims because of the increased distance between the MR probe circuitry and shim or gradient coils. Several MR probe designs for the larger horizontal bore magnets can be found in the literature $(29,35)$.

A drawing of a generalized perfusion loop for a BAL is shown in Fig. 2 with the various components. Everything present in a standard life-support unit of a BAL system is present in the MR-compatible system, except that the perfusion lines are lengthened and the recommended heating and oxygenation are in parallel and near the magnet. There are two benefits to this: (i) the perfusion medium can be maintained at room temperature, inhibiting bacterial growth and the degradation of medium components, such as glutamine; and (ii) there is no outgassing in the bioreactor as a result of a decrease in solubility of oxygen caused by an increase in temperature in the magnet. An MR-compatible, autoclaveable gas exchange/heater module has been described that solves these issues (36). It is very important to place a bubble trap just before the medium line enters the bore at the top of the vertical bore magnet. Bubbles can become trapped inside the BAL and destroy spectral quality because of the enormous magnetic susceptibility effects caused by the air-water interface.

Figure 2 shows the in-line oxygen electrodes to monitor oxygen consumption. This is difficult with the Clark electrode for long-term tissue engineering studies as the membrane may become fouled and, if one monitors medium oxygen levels periodically via ' $\mathrm{T}$ ' connections throughout the study period, these are potential sources of contamination. If oxygen consumption is desired, a noninvasive fluorescent oxygen sensor is recommended - they do not consume oxygen during the measurement, unlike the Clark electrode, which becomes important for small volumes. At the very least, it is essential to measure the drop in oxygen concentration from the gas exchange module down the water-jacketed line to the bioreactor, as polyethylene tubing is extremely permeable to oxygen and will diffuse into the water-jacket fluid, typically decreasing the oxygen concentration once entering the BAL. Autoclaveable fluorinated ethylenepropylene (FEPT) tubing (0.0313-in inner diameter) is recommended, which is less oxygen permeable than polyethylene tubing. A $20 \%$ drop from $95 \%$ oxygen exiting the gas exchange module and traveling down a 3-ft length of tubing at $3 \mathrm{~mL} / \mathrm{min}$ and $37^{\circ} \mathrm{C}$ to the bioreactor is not unexpected. Once this drop in oxygen concentration is measured and the flow rate remains constant, one can assume this value for the length of the experiment.

The fluidized-bed BAL (Fig. 1A, C), described below in the 'entrapment bioreactor' category, fits inside a standard 10-mm MR probe and is lowered from the top of the magnet, as is typical for vertical bore magnets, and uses the spectrometer VT system to help maintain the temperature [see ref. (32)] (Fig. 2). However, for larger oddly shaped BALs, the MR probe can be built around the bioreactor, and the MR probe-bioreactor assembly is inserted into the magnet from the bottom. Figure $3 \mathrm{~A}$ shows the assembly being inserted into an unshielded wide-bore 400-MHz NMR spectrometer with the life-support accessories on a cart at a distance (Fig. 3A, foreground). With shielded magnets, the cart can be right next to the magnet and the lines much shorter. A simple Helmholtz coil can be constructed using flexible circuitry described previously (29) and shown in Fig. 3B. A dual,

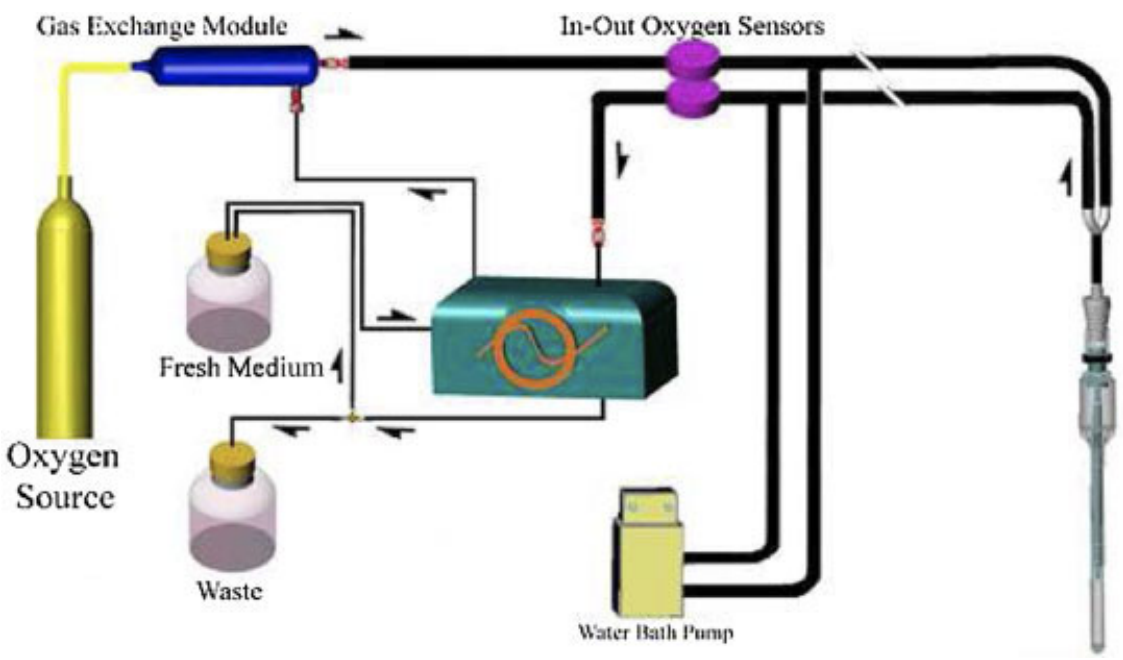

Figure 2. Generic bioreactor life-support loop and bioreactor. Block diagram of the bioreactor loop showing the major components and the gas exchange module that heats and oxygenates the medium simultaneously (12), so that it can be maintained at room temperature. 
(A)

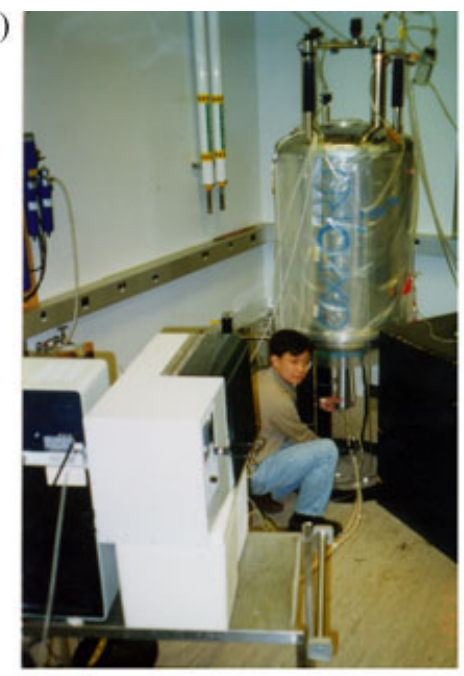

(D)

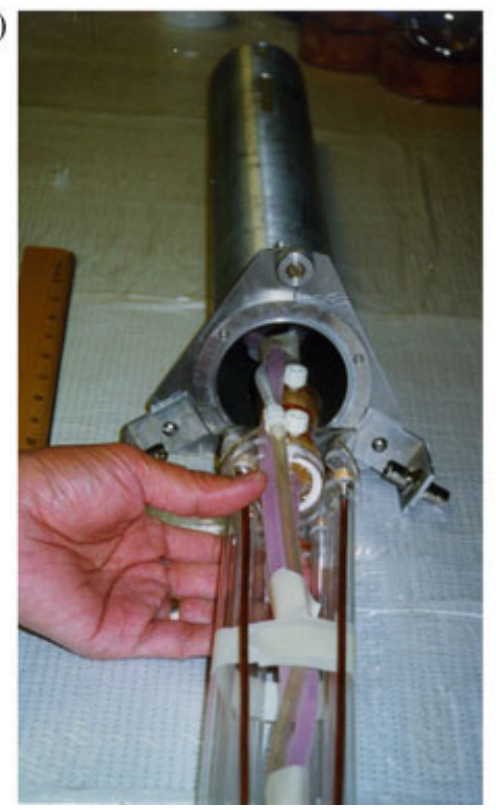

(B)

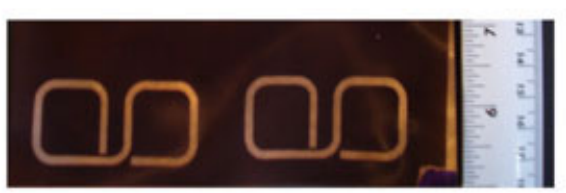

(C)

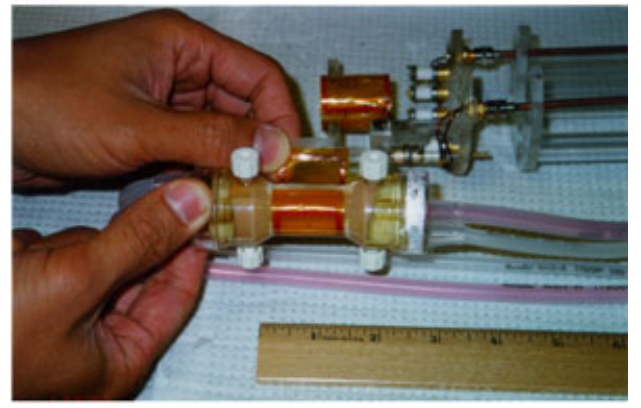

(E)

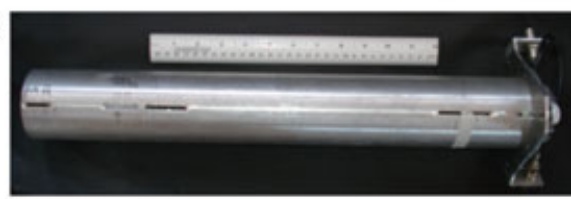

(F)

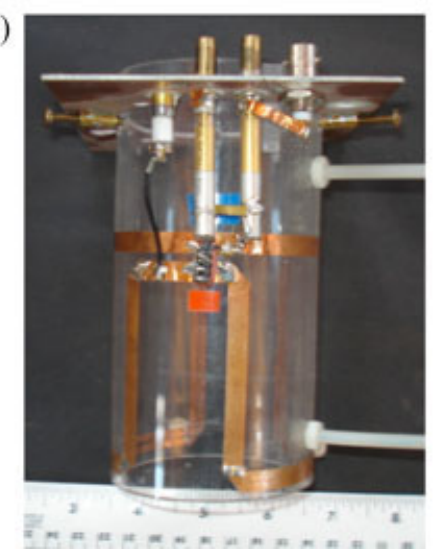

Figure 3. MR compatibilization of bioartificial livers (BALs), showing the insertion of the assembly into the bottom of a wide-bore 400-MHz NMR spectrometer (A) and the photo-etched flexible Helmholtz copper coils (B) that are wrapped around the hybrid hollow fiber-microcarrier BAL to serve as the low-frequency coil, increasing the filling factor; this is then inserted into the outer high band coil wrapped on two semicircular glass cylinders and soldered to the circuit in the background (C). The BAL-loop-MR probe-cradle assembly is inserted into an aluminum radiofrequency (RF) shield (D and E), or a larger imaging probe, either commercial or home-built, can be used to obtain images, as shown by the ${ }^{1} \mathrm{H} /{ }^{23} \mathrm{Na} \mathrm{NMR}$ probe (F).

orthogonally placed Helmholtz coil permits a broad-band MR probe to be made around the BAL (Fig. 3 C), wherein the inner coil is attached to a circuit tuned to ${ }^{13} \mathrm{C}$ and ${ }^{31} \mathrm{P}$ frequencies (Fig. 3C, foreground) and the outer coil is attached to a circuit tuned to ${ }^{1} \mathrm{H}$ frequency (Fig. 3C, background) for decoupling and MRI. As a result of the proximity of the room temperature shims to the MR coil in vertical bore magnets, the BAL-MR probe cradle assembly is inserted into an aluminum RF shield (Fig. 3D), which runs the length of the magnet and is grounded to the magnet at its base. It also contains a connection, in this case Bayonet Neill-Concelman (BNC) (Fig. 3E), for coaxial cable(s) leading to the preamplifier. Horizontal magnets provide more space, permitting the BAL to be inserted into a large-bore ${ }^{1} \mathrm{H}$ MR probe (Fig. $3 F$ ).

\section{MULTINUCLEAR MRS OF A HYBRID HOLLOW FIBER-MICROCARRIER BAL}

Ugurbil et al. (37) first introduced microcarrier beads, whereby mouse embryo fibroblasts were grown on the surface of polymeric beads and placed in an MR-compatible perfusion chamber for study. With microcarriers and encapsulates, a packed bed is perfused. In the former, the global density is hindered because the cells only attach to the outside of the microcarrier, with microcarrier material void of cell signal, resulting in a low cell number per unit volume of microcarrier, and thus SNR is adversely affected (Table 1). Membrane bioreactors typically separate the cells from the flowing perfusion solution by 
permeable membranes, allowing the diffusion of nutrients and waste, but restraining the cells in the bioreactor. A hollow fiber bioreactor is a membrane system that consists of a bundle of permeable hollow fibers running axially (14) or radially (38) through the housing, typically capped by epoxy or thermal welding, with medium or gas typically traveling through the fiber lumen (39). The hybrid hollow fiber-microcarrier bioreactor (Genespan Inc., Bothell, WA, USA) is shown in Fig. 1A, wherein there is an annular ring of polyethylene oxygenation hollow fibers (300- $\mu \mathrm{m}$ outer diameter) and a central bundle of nutrient delivery cellulose acetate hollow fibers (3-mm outer diameter) (Fig. 1B, C), and microcarriers rest in between the two bundles and rotate $120^{\circ}$ on a rocker bed. Figure $4 \mathrm{~A}$ shows the bioreactor being inoculated via the side port with hepatocyte-coated dextran microcarriers and, in the three experiments presented in Fig. $4 \mathrm{~A}$, a total of 500 million rat hepatocytes were inoculated in each bioreactor.

(A)

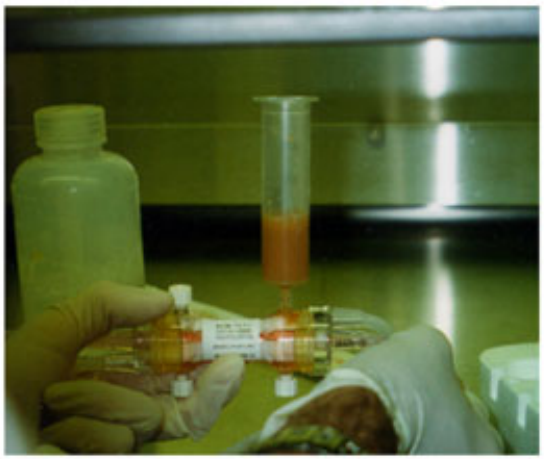

(C) Media Analysis
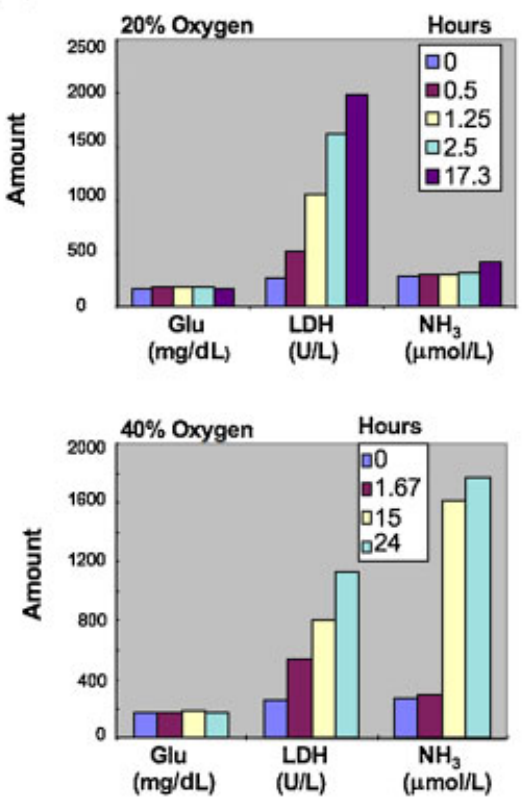

\section{Viability by ${ }^{31} \mathrm{P}$ MRS}

The ${ }^{31} \mathrm{P}$ MR spectrum contains viability information by monitoring $\beta$-nucleotide triphosphate ( $\beta$-NTP) over time (29), which is approximately $80 \%$ adenosine triphosphate (ATP) in liver (40). The first ${ }^{31} \mathrm{P}$ MRS cell studies of hollow fiber bioreactors were used not only to monitor cell viability, but also cell growth within the opaque material of these bioreactors $(41,42)$. Dale and Gillies (1) subsequently refined the MR-compatible design to optimize global density (i.e. the percentage of volume within the MR probe consisting of cell mass), and enabled cell culture for many months to reach tissue densities. The first MR-compatible hollow fiber BAL was published by Macdonald et al. (29). As an example of how ${ }^{31}$ P MRS can be used in the development of BALs, data from experiments with a hybrid hollow fiber-microcarrier bioreactor with various oxygen concentrations and flow configuration are given below. In general, oxygen is always the rate-limiting nutrient in BALs and,

(B)
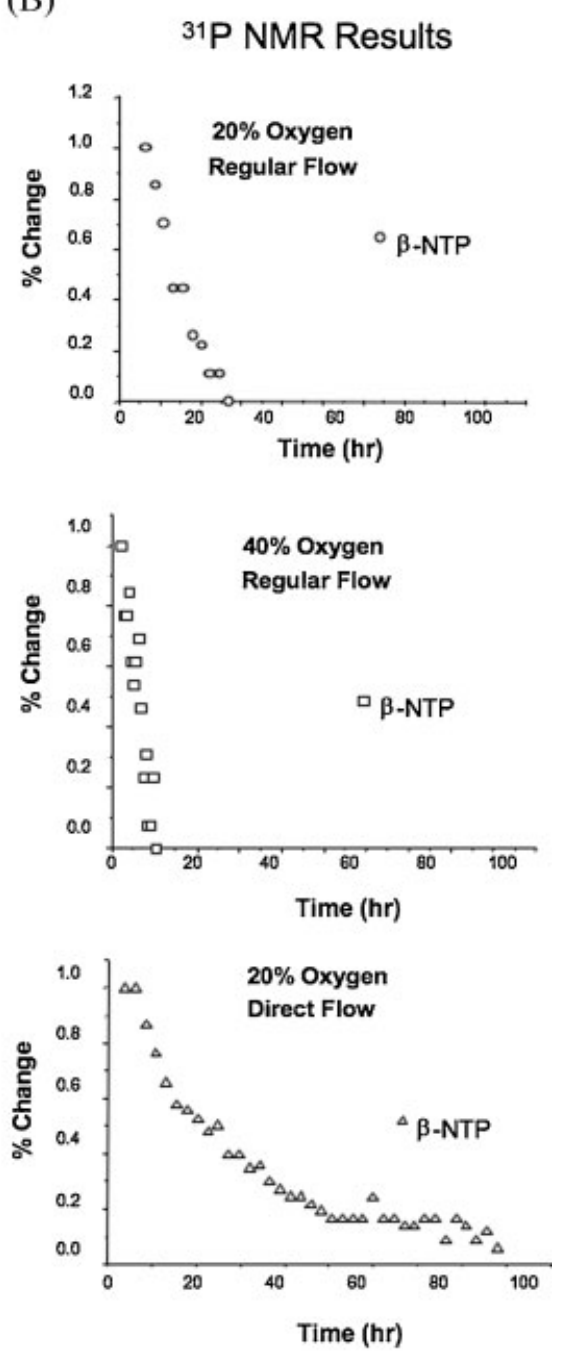

Figure 4. (A) Inoculation of the hybrid hollow fiber bioreactor with hepatocytes mixed with microcarriers. (B) Graphs of the percentage change in $\beta$-nucleotide triphosphate ( $\beta$-NTP) relative to $\beta$-NTP from the first spectrum of hepatocytes perfused with medium subjected to $20 \%$ and $40 \%$ oxygen, and $20 \%$ oxygen but directly perfused through the cell compartment. (C) Analysis of the medium components after $20 \%$ and $40 \%$ oxygen treatments with a regular flow configuration, showing the increase in lactate dehydrogenase (LDH), validating that the decrease in $\beta$-NTP is a result of cell death (glucose and ammonia are also quantified). 
typically, a distance beyond approximately $200 \mu \mathrm{m}$ is considered to be hypoxic $(15,29,43)$, while physiolgically this distance ranges from 20-60 $\mu \mathrm{m}(15,44)$. In order to address this issue, the $10-\mathrm{cm}$ space between oxygen and nutrient fibers (Fig. 1B) was subjected to $30-60 \mathrm{~Hz} 120^{\circ}$ rotation on a rocking stand, and microcarriers containing hepatocytes were inoculated into this space (Fig. 1C).

Two oxygen concentrations were tested in the hybrid hollow fiber-microcarrier BAL: $20 \%$ and $40 \%$ oxygen with $5 \%$ carbon dioxide with the remainder nitrogen. The bioreactor was taken off the rocker and placed in the MR probe (Fig. $3 \mathrm{C}$ ), and ${ }^{31} \mathrm{P} M R$ spectra were obtained immediately after inoculation. Figure $4 \mathrm{~B}$ shows the time courses of the $\beta$-NTP peak area relative to the $\beta$-NTP peak area obtained in the first spectrum, expressed as the percentage change. In all cases, $\beta$-NTP decreased precipitously with $20 \%$ and $40 \%$ oxygen. Figure $4 \mathrm{C}$ shows the results of the analysis of medium components, illustrating the increase in lactate dehydrogenase in both $20 \%$ and $40 \%$ oxygen treatments, confirming cell death. One of the main problems with hepatocytes is that the cell surface adhesion proteins, such as cadherin, cause hepatocytes to aggregate and, indeed, this is the mechanism by which liver spheroids are produced (31). As the hepatocytes are on the surface of the collagen-coated microcarriers, and although the bioreactor is constantly rotated by $120^{\circ}$ on a rocker (Fig. 1C, bottom) at a period of $30 \mathrm{~Hz}$, the hepatocytes still gel in the bioreactor. The gel was so solid that, in a third experiment, the side port was perfused and a small portion of hepatocytes near the perfusion channel remained viable for 3 days (Fig. 4B, bottom graph), completely eliminating the usefulness of the hollow fiber bioreactor. In fact, this gelling phenomenon makes it impossible to suspend hepatocytecovered microcarriers in a fluidized-bed bioreactor without gelling (Table 1), and has required alginate/agarose encapsulation methods to be employed to avoid aggregation phenomena (see below)

\section{Quantification of drug metabolic activity and oxygen distribution by ${ }^{19} \mathrm{~F}$ MRS}

A common functional assay for hepatocytes is the administration of a metabolic probe, such as ethoxycoumarin, lidocaine or benzodiazepine, to quantify cytochrome P450 activity (45). ${ }^{19} \mathrm{~F}$ MRS has been used to perform similar measurements in whole animals $(46,47)$ and in cells $(48)$. Similar to a previous ${ }^{19} \mathrm{~F}$ MRS study of substituted fluorinated phenols (49) a cytochrome P450 (IA1 and IIB1) substrate, 4-fluoroacetanilide, was given to rats and the urinary metabolites were analyzed (50). 4-Fluoroacetanilide is an ideal metabolic probe to test the metabolic activity of MR-compatible BALs, because nearly all major drug-metabolizing enzymes can be probed (50), including acetylation/deacetylation, flavo-mononamine oxidase, cytochrome P450s and all of the major phase II conjugating pathways (i.e. glucuronidation, sulfation and gluthionylation), the latter requiring the analysis of media streams. The relative distribution of fluorinated metabolites in the MR-compatible BAL can be compared with that in whole organisms (50). Scarfe et al. (50) found, in rat, that 4-fluoroacetanilide was metabolized by cytochrome P450 to 4-fluoro-2-hydroxyacetanilide (ortho-hydroxy rather than para-hydroxy acetaminophen) and subsequently conjugated to glucuronide, whereas two-fold more was metabolized by deacetylation to 4-fluoroaniline, and then ortho-hydroxylated by cytochrome $\mathrm{P} 450$, and conjugated by sulfation. This metabolic probe is well resolved in the ${ }^{19} \mathrm{~F}$ MR spectrum, with the two major metabolites, P450oxidized glucuronide and deacetylated-sulfate conjugates, resonating at $-4.2 \mathrm{ppm}$ and $+6.8-\mathrm{ppm}$, respectively from the parent compound. This permits sufficient resolution to monitor drug metabolism kinetics noninvasively, as shown in Fig. 5, which demonstrates an increase in the peak upfield from the parent.

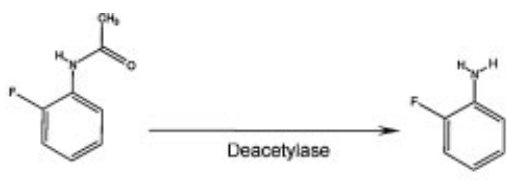

2-Fluoroacetanilide Time Course

Deacetylated Metabolite

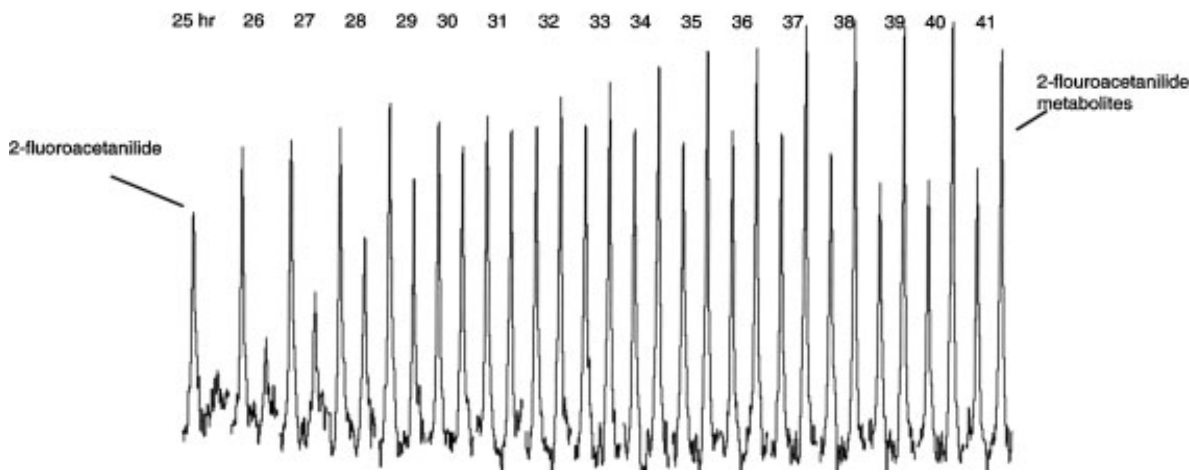

Figure 5. ${ }^{19} \mathrm{~F}$ MR spectral time course of the metabolic probe, 2-fluoroacetanilide, and its metabolite, 2-fluoroaniline, with their structures and metabolic pathway (top). Each spectrum required $1 \mathrm{~h}$ to acquire. The hybrid hollow fiber was used and inoculated with microcarriers coated with rat hepatocytes and perfused with Dulbecco's modified Eagle's medium oxygenated with $20 \%$ oxygen : $5 \%$ carbon dioxide : $75 \%$ nitrogen. The fluorinated metabolic probe was added to the medium after $24 \mathrm{~h}$ of ${ }^{31} \mathrm{P}$ acquisition, as shown in Fig. $4 \mathrm{~B}$ for $20 \%$ oxygen treatment (top graph); therefore, the time course starts at $25 \mathrm{~h}$ post-inoculation. 
To demonstrate the in situ analysis of drug metabolism in an MR-compatible BAL, 2-fluoroacetanilide, rather than 4-fluoroacetanilide, was used, because this compound could be metabolized by cytochrome P450 directly to the fluorinated acetaminophen analog, 2-fluoro-5-hydroxyacetanalide (parahydroxy rather than ortho-hydroxy). The metabolism of 2-fluoroacetanilide was monitored by ${ }^{19} \mathrm{~F}$ MRS in the hollow fiber-microcarrier BAL, shown in Fig. 1A, using 20\% oxygen. Although the medium was oxygenated with $20 \%$ oxygen and the hepatocytes were in the process of dying, as supported by the ${ }^{31} \mathrm{P}$ MR spectral course shown in Fig. 4B (top graph), they still metabolized the substrate (Fig. 5). After $24 \mathrm{~h}$ of ${ }^{31} \mathrm{P}$ time course (Fig. 4B, top graph), 2-fluoroacetanilide was added to the perfusion medium consisting of hormonally defined Kubota's medium (33), and $1-\mathrm{h}{ }^{19} \mathrm{~F}$ MR spectra were obtained using the outer high band coil (Fig. 3C, in background), which required about $3-4 \mathrm{~h}$ to equilibrate with the gelled tissue mass (Fig. 5 - time to plateau of parent) in the cell compartment (Fig. 1B, left drawing). The fluorinated metabolite initially increased over $10 \mathrm{~h}$ and then leveled off, and the parent did not change, indicating death of the BAL culture. Interestingly, deacetylase activity continued for at least $10-12 \mathrm{~h}$ (Fig. 5 - time to plateau of metabolite) after $\beta$-NTP was no longer detectable (Fig. 4B, top graph), as supported by the increase in the peak upfield from the parent (Fig. 5). As expected, the downfield peak representing direct cytochrome P450 hydroxylation was not detected (50). The proportion or distribution of hepatocytes within the BAL metabolizing the compound was not determined. This is the first demonstration of the use of ${ }^{19} \mathrm{~F}$ MRS to measure drug metabolic activity within BALs, and one could fit the ${ }^{19} \mathrm{~F}$ MRS time course to the appropriate function in order to derive the kinetic rate constants for the respective metabolic pathways, with periodic testing over weeks and months of culture to determine long-term function.

${ }^{19} \mathrm{~F} \mathrm{MRI}$ has been demonstrated to image oxygen concentrations within a coaxial hollow fiber bioreactor (51), but has not yet been performed with BALs. This method perfuses a perfluorinated hydrocarbon into the bioreactor, and $T_{1}$ maps are obtained across the bioreactor in order to obtain the oxygen concentration. One problem with this method is that perfluorinated hydrocarbons are highly lipophilic $(52,53)$ and thus are retained in the bioreactor, and they are peroxisome proliferators (54).

\section{${ }^{1}$ H MRI OF A HOLLOW FIBER MULTICOAXIAL BAL}

MRS and MRI in the field of tissue engineering have primarily studied membrane BALs, in the form of a hollow fiber bioreactor. Hollow fiber bioreactors typically possess sufficient mass transfer, but relatively poor global densities, and thus poor SNR for MRS (Table 1), because much of the sensitive volume within the MR probe is filled with bioreactor material. A coaxial hollow fiber bioreactor is composed of a fiber within a fiber (Fig. 1B, middle drawing) and can replicate the dimensions of the liver lobule (15). The first published MRI studies of a hollow fiber bioreactor were of a coaxial design to investigate fiber concentricity and cell distribution (55). They found that, although central fibers were constructed asymmetrically, the cell culture of a mouse hybridoma cell line apparently centered the fiber. As the bioreactor was opaque and composed of polypropylene, MRI was the only method to observe the soft tissue cultured cells in the annular space between fibers (Fig. 1B, middle drawing).

\section{BAL integrity and quality assurance}

Simple $T_{1}$-weighted or $T_{2}$-weighted ${ }^{1} \mathrm{H}$ MRI can be extremely useful in determining BAL integrity, with several specific examples of the use of ${ }^{1} \mathrm{H}$ MRI with hollow fiber bioreactors. For example, polypropylene hollow fibers require wetting by ethanol in order to become permeable to water. This is called the 'wetting' process and, if not performed correctly, there will be no flow across these fibers and the cells required to be perfused by these dry fibers will die as a result of a lack of diffusion of medium and oxygen. $T_{2}$-weighted images reveal hypointense regions, which identify the dry fibers. The reader is referred to Macdonald et al. (29) to show how $T_{2}$-weighted MRI was used to monitor BAL wetted fibers, and how the wetting process was modified to ensure that all fibers were thoroughly wetted. In a similar study with cell lines, diffusion-weighted MRI was employed to monitor fiber breach in extremely fragile hollow fibers made of cellulose acetate (56) using an MR-compatible bioreactor by Gillies et al. (14). In this case, there was a dramatic change in diffusion-weighted MRI after the cells had reached confluency, and the pressure from the growing cell mass on the fiber walls caused them to break (56).

Quality assurance of the bioreactor is also an important application of simple $T_{1}$-weighted or $T_{2}$-weighted ${ }^{1} \mathrm{H}$ MRI. Figure $6 \mathrm{~A}$ shows transaxial $T_{2}$-weighted ${ }^{1} \mathrm{H}$ MRI of two prototypes containing 18 (left) and 40 (right) fiber pairs (29). The images define the three compartments of the coaxial bioreactor: (i) the intracapillary compartment (ICC); (ii) the annular space; and (iii) ECC. MRI was not only used to determine the global densities of the two prototypes, employing MRI area analysis of the annular compartment divided by the total cross-sectional area of the

\section{(A)}

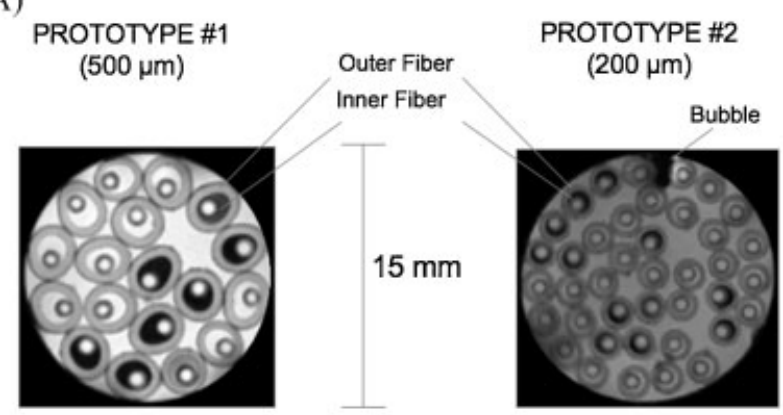

(B)

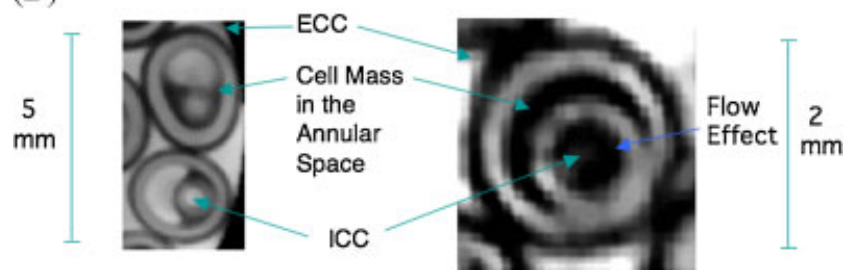

Figure 6. (A) Transaxial $T_{2}$-weighted MRI of two coaxial hollow fiber prototypes, with 18 (left) and 40 (right) fiber pairs with diffusion distances of 500 and $200 \mu \mathrm{m}$, respectively. (B) The enlargement of a partially (left) and completely (right) filled fiber pair from the 18- and 40-fiber pair prototypes. The effect of flow results in hypointensity in the intracapillary compartment (ICC) ( $\mathrm{B}$, right image). Modifications of images from Macdonald et al. (29). 
bioreactor (diameter $\times \pi$ ), but also for quality assurance. Both prototypes are made of polypropylene and are reused after extensive acid/base washing of the cell compartments. There is residual cellular material filling the annular space, represented by hypointense regions in MRI (Fig. 6A) within the cell compartment (see Fig. 1B, middle drawing). Both images were obtained the end of an experiment after cells were forced out of the annular space by pressure, via syringe, showing that the 18- and 40-fiber pair prototypes had 7 and 12 fiber pairs containing hepatocyte debris respectively, and required more stringent cleaning with $2 \mathrm{~m} \mathrm{NaOH}$ (29). In addition, there is a bubble detected on the top of the image of the 40-fiber pair prototype. This occurred as a result of incomplete filling of the ECC (see Fig. 1B, middle drawing); cellular material or air lodges in this location because the bioreactor is set on its side, and this would be the top of the bioreactor if it were on its side in a horizontal magnet (29).

\section{Inoculation efficiency}

One of the most difficult and important aspects of BAL operation is the inoculation efficiency and distribution. Opaque bioreactors do not permit one to observe the distribution of inoculated cells. Macdonald et al. (29) were the first to use ${ }^{1} \mathrm{H}$ MRI to determine BAL inoculation efficiency (i.e. percentage of bioreactor cell compartment filled with cell inoculate) using the coaxial hollow fiber bioreactor. Figure $6 \mathrm{~A}$ shows two coaxial bioreactor prototypes with different diffusion distances, which were compared to determine the effect of diffusion distance on hepatocyte viability. As expected, when the 18-fiber pair prototype was $100 \%$ inoculated (i.e. the entire cell space was filled), the diffusion distance was too large and the hepatocytes died, as monitored by ${ }^{31} \mathrm{P}$ (29). However, when the 18-fiber pair prototype was partially filled with hepatocytes, as shown in Fig. 6B (left image), they survived (29). Indeed, the diffusion distance was calculated for the two prototypes from similar images shown in Fig. 6B.

In short, if not for MRI, the evaluation of the inoculation efficiency would be extremely time consuming, and any invasive method may void the cell compartment making it very difficult to retain confidence in the results. Given that the bioreactor is made of nonmagnetic materials, MRI is the best method for the evaluation of bioreactor inoculation efficiency and, as demonstrated in the example in Fig. 6, resulted in design modifications.

\section{Flow dynamics}

Hammer et al. (57) published the first use of ${ }^{1} \mathrm{H}$ MRI to measure flow in bioreactors, which was performed on a hollow fiber bioreactor containing no cells. Donoghue et al. (58) followed, in 1992, using velocity-encoded MRI to determine media velocity profiles in hollow fiber bioreactors without cells. Simple $T_{2}$ - and $T_{1}$-weighted MRI (29) and tailored pulse sequences (59) were first used in BALs to observe media flow. Flendrig et al. (59) modified the RF coil to tag the water prior to entering the sensitive volume of the coil, wherein the flow was hyperintense, whereas Macdonald et al. (29) simply used a standard Helmholtz coil and $T_{2}$-weighted pulse sequences. The effect of flow on $T_{2}$-weighted MRI is hypointensity, as demonstrated by comparing the MR images in Fig. 6B. A hydrodynamic study similar to that performed by Hammer et al. (57) was carried out with the multicoaxial hollow fiber design, whereby velocity-encoded ${ }^{1} \mathrm{H}$ MRI (33) was applied to determine the velocity of flow in the inner and outer media compartments (ICC and ECC, Fig. 1B, middle
(A)

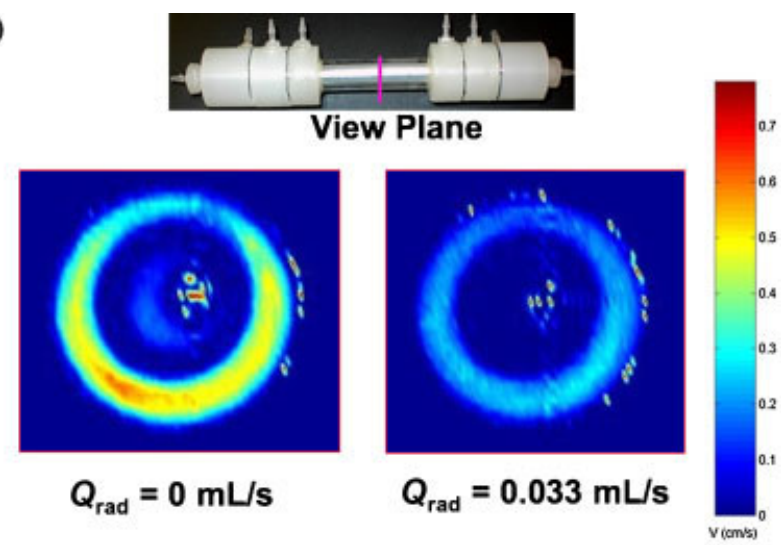

(B)

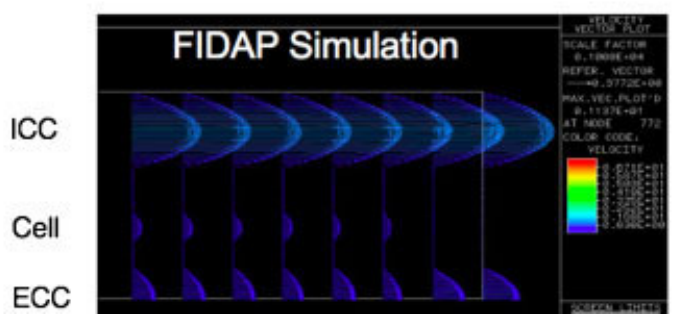

Figure 7. (A) Transaxial velocity-encoded MRI of the multicoaxial hollow fiber bioreactor (inset) at two inward radial flow rates. Note the difference in flow of the extracapillary compartment (ECC) between the two flow rates, and that the intracapillary compartment (ICC) flow is out of the range of velocities calibrated for the MRI experiment and is thus folded. The FIDAP (Fluid Dynamics Analysis Package) theoretical analysis of flow in the three compartments is given in (B). Note that the laminar nature matches the ECC and cell compartments, and predicts a higher velocity profile in the ECC. $Q_{\text {rad }}$ radial flow. Reproduced by permission from Wolfe et al. (33).

drawing), which surround the annular cell compartment, using the flow configuration shown in Fig. 1C. Figure 7A displays two velocity-encoded MR images with different radial flow rates. Specifically, radial flow was diverted from the outer fiber inward, initially with no radial flow $(0 \mathrm{~mL} / \mathrm{min})$ (Fig. 7A, left) and then at $0.33 \mathrm{~mL} / \mathrm{min}$ (Fig. 7A, right). The flow in the ICC is so fast that it is folded and outside the calibration curve of velocities for the MRI experiment, and does not fit the velocity scale in Fig. 7A. However, flow in the ECC is laminar. The difference in velocity profiles of the two MR images resulted in a radial flow rate that was the same as the measured difference between the ICC and ECC flow rates, as determined using a graduated cylinder to quantify the volumes exiting ECC and ICC (33). The velocity-encoded MRI confirmed benchtop studies showing that the outer polysulfone hollow fiber had a permeability that was two orders of magnitude higher than that of the inner fiber, and thus radial flow at physiological pressures was not possible (33). Indeed, this single finding led to the development of a second-generation bioreactor, whereby the outer, relatively impermeable polysulfone fiber was replaced with a woven vasculature that was $3 \mathrm{~mm}$ in diameter, wherein permeability could be easily modified by the weave tightness and curing process $(60,61)$.

One interesting discovery was that, with no radial flow from ECC to ICC, there was axial flow in the annular cell compartment (Fig. 1B, middle drawing) using a configuration with the ECC and ICC flows in the same direction (Fig. 1C, see flow arrows). Figure 7A (left) shows axial velocity-encoded MRI, indicating no 
radial flow, yet there is axial flow in the cell compartment. Using the hydraulic permeability of the two hollow fibers determined from benchtop studies (33), FIDAP (Fluid Dynamics Analysis Package, Fluid Dynamics International, Ltd., Taplow, UK) simulations of flow (Fig. 6B) predicted that there is axial Starling flow in the cell compartment when there is flow in the ICC, because of the two order of magnitude higher permeability of this inner hollow fiber (33). This flow diminishes axially as a result of the Starling flow effect, whereby the radial flow reverses direction and goes back into the ICC because of the drop in pressure across the ICC from inlet to outlet. This is because the pressure is higher in the ICC at the inlet than in the cell compartment, but reverses at the outlet. Of course, when the cell compartment is filled with cells, unless there are axial conduits for the flow, this axial flow will not occur in the cell compartment because of the resistance caused by the cell mass. Another observation confirmed by MRI is that the flow is laminar in the ECC and cell compartment (Fig. 6A), as predicted by the FIDAP model (Fig. 6B), but is off scale in MRI for the ICC. However, the MRI pulse sequence could be re-calibrated to include the high flow rate of the ICC on the velocity scale, but resolution for the lower flow rates will decrease, and perhaps become undetectable, given the same acquisition time. This demonstrates the type of empirical data generated by velocity-encoded ${ }^{1} \mathrm{H}$ MRI for the characterization of transport phenomena in BALs, and how they can be compared with models of flow to corroborate the results. MRI compares with other modalities, such as acoustic and laser Doppler velocimetry, because it is noninvasive with little need for BAL modification for the flow measurement. That is, BAL can be made MR-compatible (Fig. 2) and placed directly in the MRI for observation.

\section{Diffusion-weighted and fast gradient echo sequence (FAST) dynamic contrast MRI}

The first demonstration of diffusion dampening ${ }^{1} \mathrm{H}$ MR pulse sequences to monitor the cell region rather than the medium region was performed in a packed-bed bioreactor of encapsulated breast cancer cells (MCF-7) in agarose threads (62), and later in a hollow fiber bioreactor (58). As mentioned above, diffusion-weighted ${ }^{1} \mathrm{H}$ MRI was first used by Callies et al. (56) to evaluate cell growth. The theoretical limit of MRI is about $5 \mu \mathrm{m}$ (63) and, therefore, with hepatocytes ranging from 7 to $25 \mu \mathrm{m}$ $(15,31)$, it is unlikely that they will be resolved without the addition of a ferromagnetic nanoparticle contrast agent (64), which could affect the biology. An ideal application, however, is the formation of organoids (65) after isolated liver cells have been cultured for several days. Hepatocytes move with philopodia and can aggregate in extracellular matrices (66). Capuani et al. (67) used diffusion-weighted ${ }^{1} \mathrm{H}$ MRI to determine the cellular volume of hepatocytes encapsulated in alginate beads, and this would be ideal for the noninvasive monitoring of the movement and aggregation of hepatocytes into organoids within BALs; however, it has not been demonstrated. For example, Fig. 8 shows a multicoaxial BAL inoculated with a $1: 1$ ratio of Matrigel $^{\mathrm{TM}}$ : collagen type 1 with 4 million hepatocytes; another BAL was inoculated with alginate-encapsulated hepatocytes, as described by Seagle et al. (68). The BALs were maintained for 30 days, and then the coaxial fiber was extricated and $6-\mu \mathrm{m}$-thick slices were cut with a microtome and stained with live-dead dye to visualize the hepatocytes. With the Matrigel ${ }^{\mathrm{TM}}$ : collagen type 1 mixture, the hepatocytes aggregated, forming an annulus over the full length of the bioreactor (Fig. 8A1-3), whereas the alginate beads did not aggregate as strongly (Fig. 8C1-4). Diffusion-weighted ${ }^{1} \mathrm{H}$ MRI would have been an ideal modality to noninvasively monitor this aggregation process and to determine the point at which aggregation was complete.

Planchamp et al. $(35,69)$ used a hollow fiber BAL inoculated with rat hepatocytes as a model to investigate the pharmacokinetics of contrast agents employing dynamic $T_{1}$-weighted FAST. The goal was not to use MRI to determine the distribution or function of the tissue-engineered system, but rather to use BAL as a liver model to investigate the disposition and biotransport of the imaging contrast agent, gadolinium benzyl-oxypropionictetraacetate. Lanthanide metals are phagocytosed by the liver's resident macrophages, Kupffer cells, and cesium is used to eliminate or kill these cells (70). Therefore, depending on the goals and composition of the cell cultures, especially if one employs a co-culture of parenchymal and nonparenchymal cells, one should consider the potential toxic effects of gadolinium contrast agents on BAL biology prior to using dynamic contrast MRI.

\section{${ }^{31} \mathrm{P}$ AND ${ }^{13} \mathrm{C}$ MRS OF A FLUIDIZED-BED BAL CONTAINING ENCAPSULATED HEPATOCYTES}

As mentioned above, the first ${ }^{31} \mathrm{P}$ and ${ }^{13} \mathrm{C}$ MRS of an MR-compatible BAL was published over three decades ago (8). The biolimiting nutrient has always been oxygen and, in order to overcome the large diffusion distances, higher concentrations of oxygen have been used for long-term viability, as demonstrated in Fig. 4 and Table 1. Although some attempts have been made to use oxygen carriers in the encapsulation matrix to increase oxygen content (52), the incorporation of perfluorinated hydrocarbons to increase oxygen delivery to hepatocytes has been inconclusive. Perfluorinated hydrocarbons do not exhibit the Bohr effect associated with hemoglobin and, at lower oxygen tension, do not release oxygen, thereby leading to higher levels of hypoxia at low oxygen tensions than found in their absence (53). Nevertheless, their use in media is advantageous; however, nearly $60 \%$ is required in perfused organs (71), and perfluorinated hydrocarbons have toxic side effects (54). Therefore, BAL studies have focused on modulating the oxygen tension $(15,31)$. Although membrane bioreactors typically have the best mass transfer, because homogeneous diffusion distances can be maintained, they suffer from poor SNR because of insufficient global density, and often have significant bulk magnetic susceptibility that diminishes the resolution (Table 1).

One advantage of a fluidized-bed bioreactor is that the SNR is good, as the diffusion distance of a membrane bioreactor is maintained by simply keeping a homogeneous diameter, whilst thoroughly percolating the beads in the fluidized bed. Indeed, if the diameter is $0.5 \mathrm{~mm}$, this is the distance across the liver acinus, and the diffusion gradients associated with the three zones of the liver acinus can be re-created with a sphere (Fig. 1B, right drawing). For example, Fig. 9A shows a comparison of the in vivo ${ }^{31} \mathrm{P}$ MR spectra obtained from a fluidized-bed bioreactor at 9.4 T and a coaxial bioreactor at $4.7 \mathrm{~T}$; the times to acquire the ${ }^{31} \mathrm{P} \mathrm{MR}$ spectra were 30 and $60 \mathrm{~min}$, respectively. Although the field strength was halved with the hollow fiber bioreactor (4.7 vs $9.4 \mathrm{~T}$ ), the inherent $T_{2}{ }^{*}$ values in the hollow fiber bioreactor are significantly less, as a result, especially, of the magnetic 

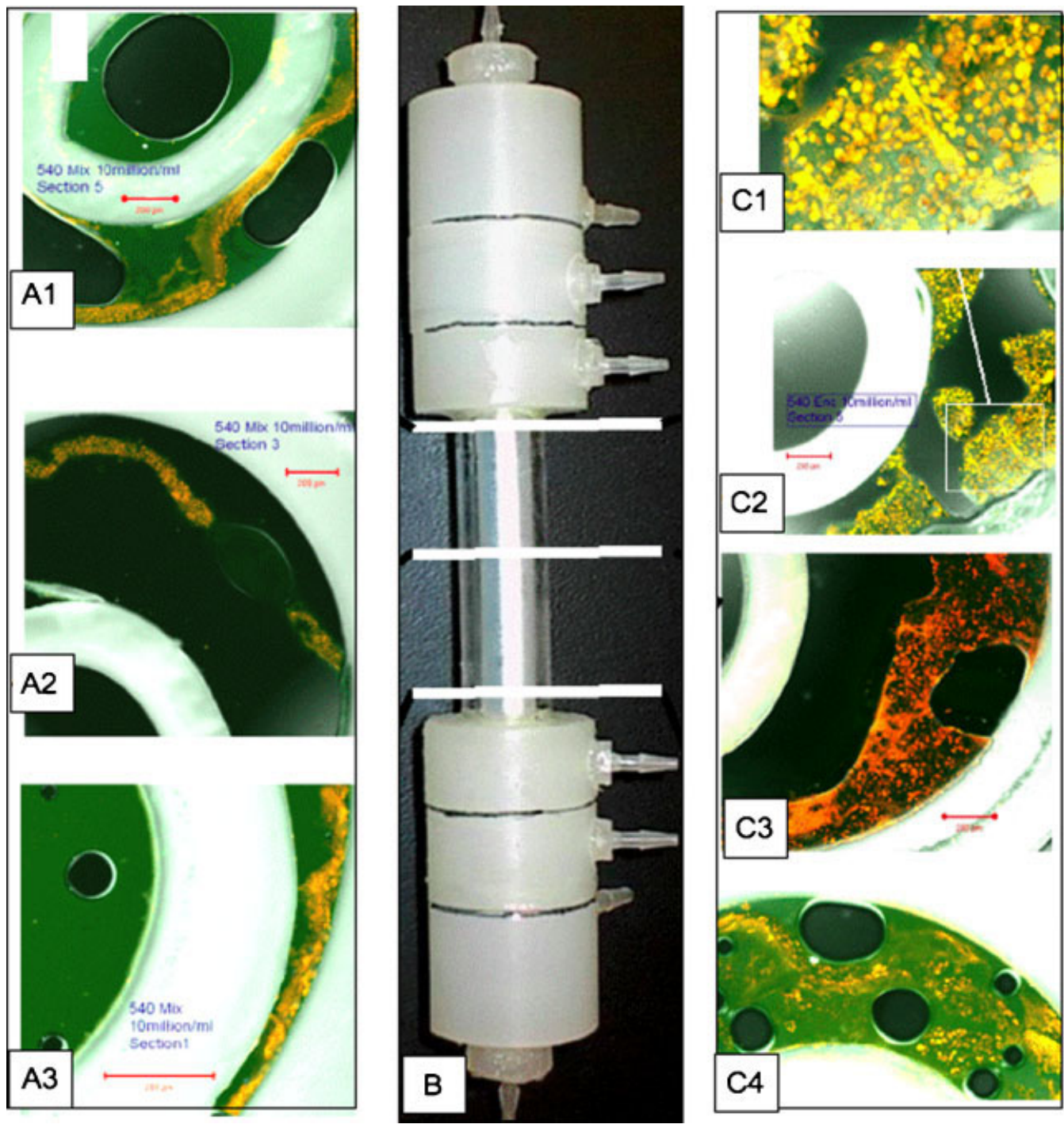

Figure 8. Confocal cross-sectional images of the coaxial bioreactor at culture day 30. (A1-A3) Cells embedded in collagen : Matrigel. (B) Generation-1 coaxial bioreactor. (C1-C4) Cells cultured with alginate : Matrigel beads. Note that the cells are aggregated in A1-A3, but not as extensively in C2-C4, except along the borders of the alginate beads (C1).

susceptibility effects. This demonstrates both the superior SNR and resolution of the fluidized bed. At a diameter of less than $700 \mu \mathrm{m}$, the alginate microspheres are solid throughout; otherwise, they can have a molten core (72).

\section{${ }^{31} \mathrm{P}$ MRS resolution, SNR, viability and $\mathrm{pH}$}

Comparison of the $\beta$-NTP time course between $20 \%$ and $35 \%$ oxygen in the perfusion medium of the fluidized-bed BAL revealed that the hepatocytes died at approximately $10 \mathrm{~h}$ after inoculation and remained viable for the course of the experiments, about $28 \mathrm{~h}$, respectively (Fig. 9B, right graph). Our data suggest that, during the first $5 \mathrm{~h}$ of perfusion with $35 \%$ oxygen, the hepatocytes recover from the hypoxic isolation process, as indicated by an increase from an acidic intracellular $\mathrm{pH} \sim 6.9$ to a physiological level of $\mathrm{pH} \sim 7.4$ (Fig. 9B), resulting in a ${ }^{31} \mathrm{P}$ MR spectrum (Fig. 9A) that is similar to the ${ }^{31} \mathrm{P}$ MR spectrum from intact liver (29). Calcium was used as gelling agent, similar to previous alginate-encapsulated BAL systems $(27,73)$, and poten- tial barium toxicities could be avoided; however, there was no difference in the ${ }^{31} \mathrm{P}$ MRS time course using barium. Although calcium is an extremely potent intracellular secondary messenger, both calcium and barium have such high affinities for carboxylate groups that they are relatively unavailable, or the encapsulates would lose integrity and disintegrate. Although the hepatocytes were maintained for $28 \mathrm{~h}$ using $35 \%$ oxygen with little change in the $\beta$-NTP peak area (Fig. 9B), they exhibited a high degree of anaerobic glycolysis not typical in liver, as indicated by the analysis of medium (68). Therefore, although in vivo ${ }^{31} \mathrm{P}$ MRS permits indirect evidence for metabolic function, in vivo ${ }^{13} \mathrm{C}$ MRS is required for noninvasive dynamic measurement of metabolic pathways.

\section{${ }^{13} \mathrm{C}$ MRS to monitor real-time metabolism}

Previous in vivo ${ }^{13} \mathrm{C}$ MRS studies of MR-compatible BALs have focused on the perturbation of intermediary metabolism as a result of xenobiotic exposure (16-18). With the advent of 
(A)
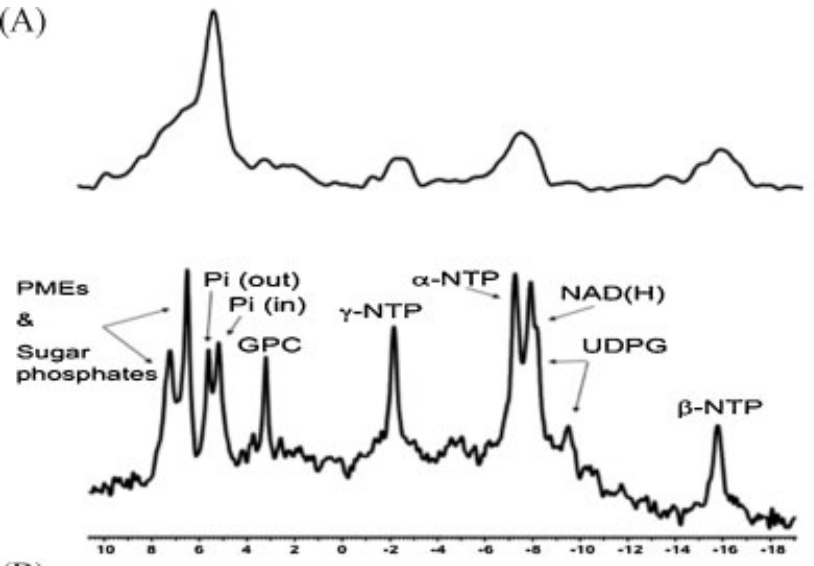

(B)
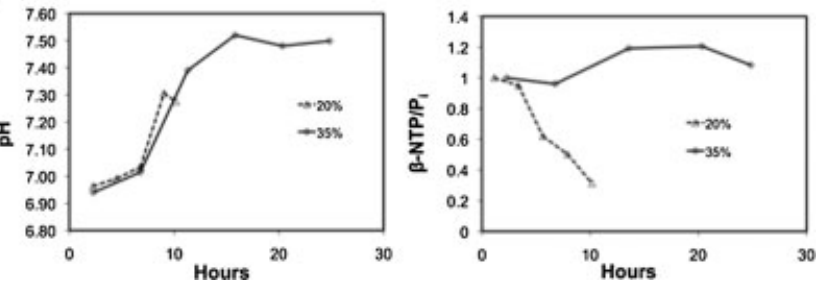

Figure 9. (A) Comparison of in vivo ${ }^{31} \mathrm{P}$ MR spectra from the hollow fiber membrane bioreactor (29) and fluidized-bed bioreactor (74) obtained in $60 \mathrm{~min}$ vs $30 \mathrm{~min}$, demonstrating the superior signal-to-noise ratio (SNR) and resolution of the fluidized-bed bioreactor. (B) Graph of intracellular $\mathrm{pH}$ (left) and $\beta$-nucleotide triphosphate ( $\beta$-NTP) (right) of rat hepatocytes perfused with medium subjected to $20 \%$ and $35 \%$ oxygen treatments. GPC, glycerophosphocholine; $\mathrm{NAD}(\mathrm{H})$, nicotinamide adenine dinucleotide; $\mathrm{Pi}$, inorganic phosphate; PME, phosphomonoester; UDPG, uridine diphosphoglucose.

perfusion of bioreactors, the ${ }^{13} \mathrm{C}$ signal from cells and medium were distinguished by the utilization of diffusion-weighted pulses (62). With the advent of perfusion of bioreactors, the ${ }^{13} \mathrm{C}$ signal from cells and medium were distinguished by the utilization of diffusion-weighted pulses (62). ${ }^{13} \mathrm{C}$ MRS studies were all performed in suspension bioreactors, primarily for metabolism studies, as mentioned above (16-18). Indeed, O'Leary et al. (75) were the first to analyze the media stream in real time by putting the bioreactor loop through the ${ }^{13} \mathrm{C}$ MR probe; the medium is called the metabolomic footprint of the cell culture and is analyzed by itself as a measure of BAL performance (68). Figure $10 \mathrm{~A}$ shows ${ }^{13} \mathrm{C}$ NMR of the fluidized-bed BAL containing alginate-encapsulated rat hepatocytes, perfused with $\mathrm{u}-{ }^{13} \mathrm{C}$-glucose, illustrating the time course of ${ }^{13} \mathrm{C}$-glucose incorporation into $\mathrm{C} 1$ of glycogen $(\sim 102 \mathrm{ppm})$. The peak reaches steady-state incorporation by about $6 \mathrm{~h}$, and the rate of glycogen synthesis can be determined by fitting the peak area to an exponential. The one problem with a fluidized-bed BAL is that pulse sequences to eliminate the large ${ }^{13} \mathrm{C}$ signal from flowing medium, such as diffusion-weighted ${ }^{1} \mathrm{H}$ MR (62), also negatively affect the signals emanating from the percolating cells. Therefore, diffusion-weighted ${ }^{13} \mathrm{C}$ MRS did not help to increase SNR and, using a one-pulse sequence, caused the $25 \mathrm{~mm}$ glucose signal to swamp the ${ }^{13} \mathrm{C}$ MR spectrum. Herein lies the inherent conundrum of in vivo MRS: replace the fluidized bed with a packed bed and redesign the bioreactor with a flow distributor (15) and containment baffles (12), so that a diffusion-weighted pulse sequence can be used for superior in vivo ${ }^{13} \mathrm{C}$ MR spectral quality, but at the expense of a higher surface velocity of medium across the encapsulate, creating nonphysiological shear conditions that could affect metabolism or destroy the encapsulate (15).

Figure $10 \mathrm{~B}$ shows the same fluidized-bed $\mathrm{BAL}$, but containing a rat hepatoma cell line, JM-1. The spectrum shows the various ${ }^{13} \mathrm{C}$ resonances representing the $\alpha$ and $\beta$ conformations of ${ }_{2}{ }^{13} \mathrm{C}$-glucose. The inset time course is the $\mathrm{C} 2$ position of lactate ( $\sim 69 \mathrm{ppm}$ ) excreted from the hepatocyte cytosol into the medium. Indeed, ${ }^{1} \mathrm{H}$ NMR of the medium and the cell extract at the end of the experiment established that this signal was almost entirely from the medium. The rate of formation is linear and can be fitted to determine lactate production and the metabolic phenotype of the hepatocytes. Normally, rat hepatocytes should not produce lactate, as liver is involved in the Cori cycle for the conversion of lactate derived from exercising muscle to glucose. Lactate production is typical of cancer cells, and thus little was detected in the ${ }^{13} \mathrm{C}$ MR spectral time course of perfused primary rat hepatocytes shown in Fig. 10A; this is an ideal functional assay of BALs and, in particular, for the determination of whether the culture is hypoxic. Indeed, this ${ }^{13} \mathrm{C}$ MRS assay is ideal for dynamically changing the oxygen content of the medium as the culture grows, or depending on the cell distribution within the bioreactor, as this can be performed essentially in real time, whilst the oxygen concentration is increased or decreased.
(A)

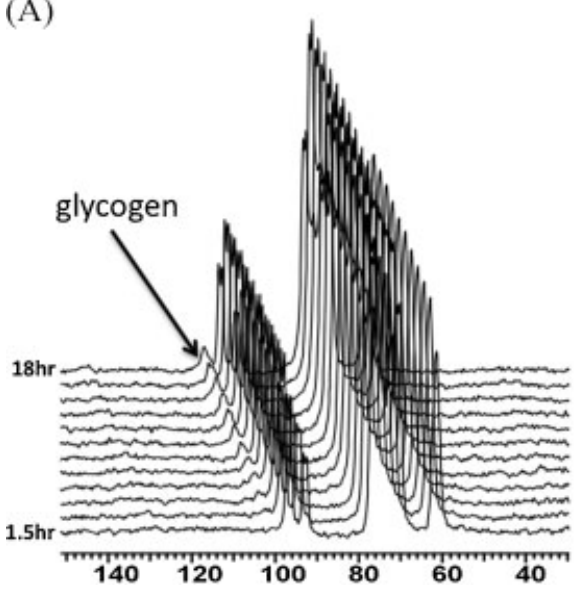

(B)

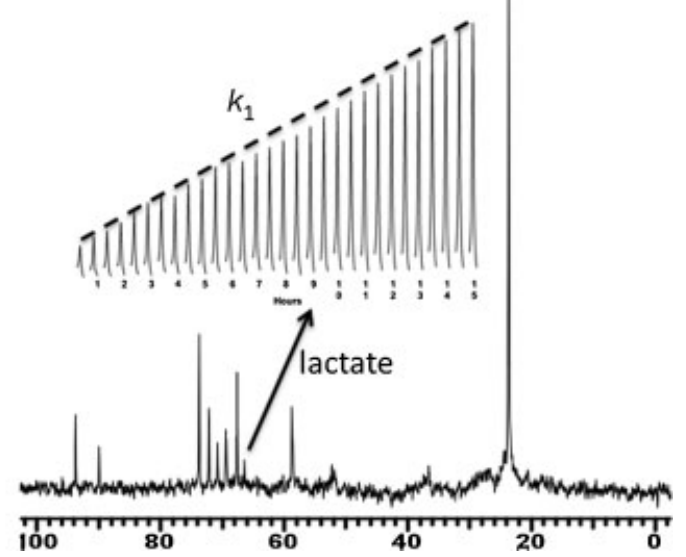

Figure 10. (A) The 18-h in vivo ${ }^{13} \mathrm{C}$ MR spectral time course of the fluidized-bed bioreactor inoculated with 500 - $\mu \mathrm{m}$-diameter electrostatically encapsulated alginate beads containing $3.5 \times 10^{7}$ rat hepatocytes $/ \mathrm{mL}$ and perfused with $25 \mathrm{~mm} \mathrm{u}^{-13} \mathrm{C}$-glucose, showing the increase in peak representing the $\mathrm{C}$-glycogen at $102 \mathrm{ppm}$. (B) In vivo ${ }^{13} \mathrm{C}$ MR spectrum of perfused alginate-encapsulated JM-1 cells and the 15-h time course of C2-lactate above the spectrum. 


\section{DISCUSSION}

Although MR-compatible BAL technology was first introduced three decades ago (8), there is still no report of a long-term device, longer than $8 \mathrm{~h}$ (Table 1), for primary hepatocytes. These early suspension and, more recently, perfused BAL studies focused on short-term xenobiotic perturbation and were not intended for long-term use (see suspension category references in Table 1). However, some have focused on the characterization of the function and imaging cell distribution for tissue engineering analysis $(29,59,69)$, and this review demonstrates the application of multinuclear MRS using ${ }^{31} \mathrm{P}$ (Figs 4 and 9 ), ${ }^{13} \mathrm{C}$ (Fig. 10), ${ }^{19} \mathrm{~F}$ (Fig. 5) and ${ }^{1} \mathrm{H}$ MRI [velocity-encoded ${ }^{1} \mathrm{H}$ MRI (Fig. 7A) and $T_{2}$-weighted ${ }^{1} \mathrm{H}$ MRI (Fig. 6)]. The integrity and inoculation efficiency of BAL have been determined using diffusion-weighted ${ }^{1} \mathrm{H}$ MRI and $T_{2}$-weighted MRI, and dynamic contrast FAST ${ }^{1} \mathrm{H}$ MRI has been applied to determine the kinetics of these agents on liver using a hollow fiber BAL as a model (69).

To recap, Table 1 shows a summary of the various MRS/MRI metrics for the four categories of bioreactor: (i) suspension (8); (ii) entrapment (3,9); (iii) microcarriers $(4,13)$; and (iv) membrane (14). The suspension bioreactor suffers the worse mass transfer, being packed in a 5-mm NMR tube with a diffusion distance of approximately $30 \mathrm{~mm}$ from the cell mass surface to the bottom of the NMR tube $(8,16-18)$. However, the suspension BAL has the best global density, resulting in the best SNR and excellent spectral resolution and line shape (Table 1). In addition, few perfused MR-compatible BAL studies used air, or $20 \%$ oxygen $(26,59)$ or $95 \%$ oxygen (19-23,27,29,69). From our results, $20 \%$ oxygen did not permit full recovery of $\beta$-NTP (Fig. 9B). Hepatocytes require relatively large amounts of oxygen. The oxygen buffering capacity of hemoglobin, which increases the oxygen concentration by nearly two orders of magnitude, maintains an oxygen gradient across the acinus of $8 \%$ to $3 \%$ (high to low) where blood exits the liver, and this is lacking in BAL perfusion systems (15).

The agarose thread encapsulation BAL (19-25) and the packed-bed bioreactor (26-28) exhibit sufficient ${ }^{31} \mathrm{P}$ MRS SNR and spectral resolution within $15 \mathrm{~min}$. However, channels can form with gel threads and a packed-bed bioreactor (15). In addition, the diffusion distance is larger than the desired $0.2 \mathrm{~mm}(26-28)$, or the diffusion distance is sufficient, but $95 \%$ oxygen was used (19-25). The MR-compatible BAL study, using the two coaxial BAL prototypes shown in Fig. 6A, compared diffusion distances of 0.2 and $0.5 \mathrm{~mm}$, and found that only the former bioreactor maintained viability (29). Other studies have shown that BALs surviving with $20 \%$ oxygen require diffusion distances of less than $200 \mu \mathrm{m}(43,76-79)$. Therefore, it may have been necessary to use $95 \%$ oxygen with these MR-compatible packed-bed alginate encapsulate bioreactors (26-28), but not the agarose gel threads (Table 1). Indeed, BALs with $95 \%$ oxygenation of the medium have survived for weeks $(15,80,81)$. Hepatocytes that survive the initial hyperoxic stress will move within the bioreactors to a region of optimal oxygen concentration via philopodia (82), and as demonstrated in the long-term cultures in Fig. 8. For example, rat hepatocytes grown in a multicoaxial bioreactor (33) have been shown to migrate and form organoid structures at an optimal distance from the oxygen sources after several days of culture (77), as illustrated in Fig. 8. Diffusion-weighted ${ }^{1} \mathrm{H}$ MRI would be ideal for monitoring this phenomenon.

Although the MR-compatible coaxial hollow fiber bioreactor design maintains a homogeneous diffusion distance and has been shown to maintain hepatocyte viability for weeks (77), the relative amount of biomass vs bioreactor/medium, or the global density (31), is much less than in entrapment and suspension bioreactors. Therefore, the temporal MR spectral resolution is relatively poor (29), generating inferior kinetic data (Table 1). However, if the goal is to monitor long-term liver function on a daily basis, typical of tissue engineering studies, in vivo ${ }^{13} \mathrm{C} /{ }^{31} \mathrm{P} /{ }^{19} \mathrm{~F}$ spectra, which require several hours to acquire, would be sufficient to achieve this goal, and much less laborious for a comparable temporal resolution when compared with high-resolution MRS analysis of media streams (68). To increase the SNR of the ${ }^{19} \mathrm{~F}$ MRS assay of phase I and phase II drug metabolism, described in Fig. 5, or the in vivo ${ }^{13} \mathrm{C}$ MRS quantification of the lactate production rate, described in Fig. 10B, the volume of medium could be minimized during the MRS measurement to increase the concentration of drugs or metabolites in the medium. For example, if the volume of medium was decreased by half, the medium lactate concentration would double, resulting in a four-fold increase in SNR for a given time.

There has never been an MR-compatible BAL reported that utilizes liver cells with microcarriers, and this is the first report of such a BAL, described in Figs $1 A$ and 4. Although, the first BAL used in clinical trials (83) employed hepatocytes on microcarriers in the initial development to increase function, they were inoculated in a hollow fiber bioreactor with a diffusion distance close to the $0.2-\mathrm{mm}$ range, unlike the 10-mm range used in this study. It was discovered that the aggregation of cells caused by cell-to-cell surface binding through calcium-dependent adhesion molecules caused large aggregates to form, resulting in large diffusion distances, as can be vaguely seen in Fig. 3C. Indeed, liver spheroid cultures depend on this aggregation effect for formation (31) and, to avoid this, spheroids (12) and microcarriers (13) are typically immobilized in a packed-bed bioreactor. The shear forces generated by the $120^{\circ}$ rotation of the hybrid hollow fiber-microcarrier BAL caused large numbers of microcarriers to aggregate.

The fluidized-bed BAL described herein (Figs $1 C$ and 2) contains sufficient biomass to yield an adequate ${ }^{31} \mathrm{P}$ MRS SNR in $30 \mathrm{~min}$ (Fig. 9A), yet maintains the maximum diffusion distance of approximately $0.2 \mathrm{~mm}$, resulting in the best characteristics of the various bioreactor types (Table 1). Oxygen studies have demonstrated that the use of $35 \%$ oxygen was required to maintain viability for the duration of the study, $28 \mathrm{~h}$ (Figs $9 \mathrm{~B}$ and $10 \mathrm{~A})$, and probably beyond. The bioreactor is versatile and designs have been constructed to fit conventional 5-mm MR probes, although a 10-mm NMR tube was used in this study. The alginate eliminates aggregation; however, it is not an extracellular matrix found in liver tissue, and therefore hepatocytes typically do not migrate as actively as they do in endogenous extracellular matrices, as shown in the confocal micrograph comparing Matrigel $^{\mathrm{TM}}$ : collagen type 1 with alginate (Fig. 8).

In short, MRS and MRI are powerful tools for noninvasive analysis in tissue engineering of the liver. Magnetic susceptibility effects and filling factors are important in the SNR of MRS and MRI quality, and some knowledge of MR probe construction can help to improve SNR, but has little effect on the innate magnetic susceptibility of the BAL design, especially designs with integral oxygenation $(59,84)$.

\section{Acknowledgements}

We thank Dr L. M. Reid for the purchase of the Genespan ${ }^{T M}$ bioreactors and hepatocyte culture supplies (R01 DK52851) and 
$\mathrm{Dr}$ R. E. London for the use of the wide-bore $400-\mathrm{MHz}$ NMR spectrometer shown in Fig. 3A. We thank Dr E. Hsu for collaborating on the flow studies of the multicoaxial bioreactor, the Center for In Vivo Microscopy of Duke University Medical Center (NCRR P41-RR05959), and the Johns Hopkins Center for Alternatives to Animal Testing, UC Toxic Substance Research and Training Program and National Institutes of Health (NIH) (F32 DK09713) for funding. We thank Stephen Wolfe for generating the FIDAP model of flow for multicoaxial bioreactor research. NIH grants (GM075941, GM38149, 1F31DK082172-01) funded the fluidized-bed bioreactor research. We appreciate the help of Alisher Holmuhamedov and Ryan Webb in constructing the bioreactors and life-support units.

\section{REFERENCES}

1. Dale BE, Gillies RJ. Nuclear magnetic resonance spectroscopy of dense cell populations for metabolic studies and bioreactor engineering: a synergistic partnership. Biotechnology, 1991; 17: 107-118.

2. Ruiz-Cabello J, Cohen JS. NMR and the study of pathological state in cells and tissues. Int. Rev. Cytol. 1993; 145: 1-63.

3. Kaplan O, Cohen JS. Metabolism of breast cancer cells as revealed by non-invasive magnetic resonance spectroscopy studies. Breast Cancer Res. Treat. 1994; 31(2-3): 285-299.

4. Szwergold BS. NMR spectroscopy of cells. Annu. Rev. Physiol. 1992; 54: 775-798.

5. Zupke C, Foy B. Nuclear magnetic resonance analysis of cell metabolism. Curr. Opin. Biotechnol. 1995; 6(2): 192-197.

6. Mancuso A, Glickson JD. Applications of NMR spectroscopy and imaging to the study of immobilised cell physiology. In Focus on Biotechnology Volume 8A: Fundamentals of Cell Immobilisation Biotechnology, Nedovic V, Wilaert R, Hofman M, Anne J (eds). Kluwer Academic Publishers: Dordrecht, Netherlands, 2004; 439-467.

7. Moon RB, Richards JH. Determination of intracellular $\mathrm{pH}$ by ${ }^{31} \mathrm{P}$ magnetic resonance. J. Biol. Chem. 1973; 248(20): 7276-7278.

8. Cohen SM, Ogawa S, Rottenberg H, Glynn P, Yamane T, Brown TR, Shulman RG. $31 \mathrm{P}$ nuclear magnetic resonance studies of isolated rat liver cells. Nature, 1978; 273(5663): 554-556.

9. Narayan KS, Moress EA, Chatham JC, Barker PB. ${ }^{31}$ P NMR of mammalian cells encapsulated in alginate gels utilizing a new phosphate-free perfusion medium. NMR Biomed. 1990; 3(1): 23-26.

10. Macdonald JM, Kurhanewicz J, Dahiya R, Espanol MT, Chang LH, Goldberg B, James TL, Narayan P. Effect of glucose and confluency on phosphorus metabolites of perfused human prostatic adenocarcinoma cells as determined by ${ }^{31} \mathrm{P}$ MRS. Magn. Reson. Med. 1993; 29(2): 244-248

11. Constantinidis I, Mukundan NE, Gamcsik MP, Sambanis A. Towards the development of a bioartificial pancreas: a ${ }^{13} \mathrm{C}$ NMR study on the effects of alginate/poly-L-lysine/alginate entrapment on glucose metabolism by beta TC3 mouse insulinoma cells. Cell Mol. Biol. (Noisy-le-Grand), 1997; 43(5): 721-729.

12. Gamcsik MP, Millis KK, Colvin OM. Noninvasive detection of elevated glutathione levels in MCF-7 cells resistant to 4-hydroperoxycyclophosphamide. Cancer Res. 1995; 55(10): 2012-2016.

13. Mancuso A, Beardsley NJ, Wehrli S, Pickup S, Matschinsky FM, Glickson JD. Real-time detection of ${ }^{13} \mathrm{C}$ NMR labeling kinetics in perfused EMT6 mouse mammary tumor cells and betaHC9 mouse insulinomas. Biotechnol. Bioeng. 2004; 87(7): 835-848.

14. Gillies RJ, Galons JP, McGovern KA, Scherer PG, Lien YH, Job C, Ratcliff R, Chapa F, Cerdan S, Dale BE. Design and application of NMR-compatible bioreactor circuits for extended perfusion of highdensity mammalian cell cultures. NMR Biomed. 1993; 6(1): 95-104.

15. Macdonald JM, Griffin J, Kubota H, Griffith L, Fair J, Reid LM. Bioartificial livers. In: Cell Encapsulation Technology and Therapeutics, Kuhtreiber W, Lanza RP, Chick WL (eds). Birkhauser Boston: Cambridge, MA, 1999; 252-286.

16. Cohen SM, Ogawa S, Shulman RG, Ardenkjaer-Larsen JH. ${ }^{13} \mathrm{C}$ NMR studies of gluconeogenesis in rat liver cells: utilization of labeled glycerol by cells from euthyroid and hyperthyroid rats. Proc. Natl. Acad. Sci. USA, 1979; 76(4): 1603-1609.
17. Irving MG, Simpson SJ, Brooks WM, Holmes RS, Doddrell DM. Application of the reverse DEPT polarization-transfer pulse sequence to monitor in vitro and in vivo metabolism of ${ }^{13} \mathrm{C}$-ethanol by ${ }^{1} \mathrm{H}-\mathrm{NMR}$ spectroscopy. Int. J. Biochem. 1985; 17(4): 471-478.

18. Nicholson JK, Timbrell JA, Bales JR, Sadler PJ. A high resolution proton nuclear magnetic resonance approach to the study of hepatocyte and drug metabolism. Application to acetaminophen. Mol. Pharmacol. 1985; 27(6): 634-643.

19. Gasbarrini $A$, Borle $A B$, Caraceni $P$, Colantoni $A$, Farghali $H$, Trevisani $F$, Bernardi M, Van Thiel DH. Effect of ethanol on adenosine triphosphate, cytosolic free calcium, and cell injury in rat hepatocytes. Time course and effect of nutritional status. Dig. Dis. Sci. 1996; 41(11): 2204-2212.

20. Gasbarrini A, Borle AB, Farghali H, Bender C, Francavilla A, Van Thiel D. Effect of anoxia on intracellular ATP, $\mathrm{Na}^{+}, \mathrm{Ca}^{2+}, \mathrm{Mg}^{2+}$, and cytotoxicity in rat hepatocytes. J. Biol. Chem. 1992; 267(10): 6654-6663.

21. Gasbarrini A, Borle AB, Farghali $H$, Caraceni $P$, Van Thiel D. Fasting enhances the effects of anoxia on ATP, $\mathrm{Ca}^{2+}$ and cell injury in isolated rat hepatocytes. Biochim. Biophys. Acta, 1993; 1178(1): 9-19.

22. Gasbarrini A, Borle AB, Farghali H, Francavilla A, Van Thiel D. Fructose protects rat hepatocytes from anoxic injury. Effect on intracellular ATP, $\mathrm{Ca}^{2+}, \mathrm{Mg}^{2+}, \mathrm{Na}^{+}$, and pH. J. Biol. Chem. 1992; 267(11): 7545-7552.

23. Farghali H, Rossaro L, Gavaler JS, Van Thiel DH, Dowd SR, Williams DS, Ho C. 31P-NMR spectroscopy of perifused rat hepatocytes immobilized in agarose threads: application to chemical-induced hepatotoxicity. Biochim Biophys Acta. 1992; 1139(1-2): 105-114.

24. Farghali H, Sakr M, Gasbarrini A, Williams DS, Dowd SR, Ho C, Van Thiel $\mathrm{DH}$. A biochemical and ${ }^{31} \mathrm{P}-\mathrm{NMR}$ investigation of the effect of FK 506 and cyclosporine pretreatment on immobilized hepatocytes perfused with ethanol. Transplant Proc. 1991; 23(6): 2805-2808.

25. Farghali H, Williams DS, Caraceni P, Borle AB, Gasbarrini A, Gavaler J, Rilo HL, Ho C, Van Thiel DH. Effect of ethanol on energy status and intracellular calcium of Sertoli cells: a study using immobilized perfused cells. Endocrinology, 1993; 133(6): 2749-2755.

26. Miccheli A, Tomassini A, Capuani G, Di Cocco ME, Sartori E, Falasca L, Conti Devirgiliis L, Manetti C, Conti F. Energy metabolism and re-establishment of intercellular adhesion complexes of gel entrapped hepatocytes. Cytotechnology, 2000; 32(3): 219-228.

27. Falasca L, Miccheli A, Sartori E, Tomassini A, Conti Devirgiliis L. Hepatocytes entrapped in alginate gel beads and cultured in bioreactor: rapid repolarization and reconstitution of adhesion areas. Cells Tissues Organs, 2001; 168(3): 126-136.

28. Murtas S, Capuani G, Dentini M, Manetti C, Masci G, Massimi M, Miccheli A, Crescenzi V. Alginate beads as immobilization matrix for hepatocytes perfused in a bioreactor: a physico-chemical characterization. J. Biomater. Sci. Polym. Ed. 2005; 16(7): 829-846.

29. Macdonald JM, Grillo M, Schmidlin O, Tajiri DT, James TL. NMR spectroscopy and MRI investigation of a potential bioartificial liver. NMR Biomed. 1998; 11(2): 55-66.

30. Foxall DL, Cohen JS, Mitchell JB. Continuous perfusion of mammalian cells embedded in agarose gel threads. Exp. Cell Res. 1984; 154(2): 521-529.

31. Macdonald JM, Xu ASL, Kubota H, LeCluyse E, Hamilton G, Liu H, Rong YW, Moss N, Lodestro C, Luntz T, Wolfe SP, Reid LM. Liver cell culture in lineage biology. In Methods of Tissue Engineering, Atala A, Lanza R (eds). Academic Press: San Diego, CA, 2002; 151-201.

32. Keshari KR, Kurhanewicz J, Jeffries RE, Wilson DM, Dewar BJ, Van Criekinge M, Zierhut M, Vigneron DB, Macdonald JM. Hyperpolarized ${ }^{13} \mathrm{C}$ spectroscopy and an NMR-compatible bioreactor system for the investigation of real-time cellular metabolism. Magn. Reson. Med. 2010; 63(2): 322-329.

33. Wolfe SP, Hsu E, Reid LM, Macdonald JM. A novel multi-coaxial hollow fiber bioreactor for adherent cell types. Part 1: hydrodynamic studies. Biotechnol. Bioeng. 2002; 77(1): 83-90.

34. Myers R. The Radio Amateur's Handbook 13th revised edition. American Radio Relay League: Crowell Publishing, New York, 1976.

35. Planchamp C, Gex-Fabry M, Dornier C, Quadri R, Reist M, Ivancevic MK, Vallee JP, Pochon S, Terrier F, Balant L, Stieger B, Meier PJ, Pastor CM. Gd-BOPTA transport into rat hepatocytes: pharmacokinetic analysis of dynamic magnetic resonance images using a hollow-fiber bioreactor. Invest. Radiol. 2004; 39(8): 506-515.

36. Gamcsik MP, Forder JR, Millis KK, McGovern KA. A versatile oxygenator and perfusion system for magnetic resonance studies. Biotechnol Bioeng. 1996; 49(3): 348-354. 
37. Ugurbil K, Guernsey DL, Brown TR, Glynn P, Tobkes N, Edelman IS. ${ }^{31} \mathrm{P}$ NMR studies of intact anchorage-dependent mouse embryo fibroblasts. Proc. Natl. Acad. Sci. USA, 1981; 78(8): 4843-4847.

38. Catapano G, Euler M, Gaylor JDS, Gerlach J. Characterization of the distribution of matter in hybrid liver support devices where cells are cultured in a 3-D membrane network or on flat substrata. Int. J. Artif. Organs, 2001; 24(2): 102-109.

39. Cima LG, Wilke CR, Blanch HW. A theoretical and experimental evaluation of a novel radial-flow hollow fiber reactor for mammalian cell culture. Bioprocess Eng. 1990; 5: 19-30.

40. Quistorff B. Metabolic heterogeneity of liver parenchymal cells. Essays Biochem. 1990; 25: 83-136.

41. Gonzalez-Mendez R, Wemmer D, Hahn G, Wade-Jardetzky N, Jardetzky O. Continuous-flow NMR culture system for mammalian cells. Biochim. Biophys. Acta, 1982; 720(3): 274-280.

42. Hrovat M, Wade C, Hawkes S. A space-efficient assembly for NMR experiments on anchorage-dependent cells. J. Magn. Reson. 1985; 61: 409-417.

43. McClelland RE, Macdonald JM, Coger RN. Modeling $\mathrm{O}_{2}$ transport within engineered hepatic devices. Biotechnol. Bioeng. 2003; 82(1): $12-27$.

44. Macdonald JM, Schmidlin O, James TL. In vivo monitoring of hepatic glutathione in anesthetized rats by ${ }^{13} \mathrm{C}$ NMR. Magn. Reson. Med. 2002; 48(3): 430-439.

45. Richert L, Binda D, Hamilton $G$, Viollon-Abadie $C$, Alexandre $E$ Bigot-Lasserre D, Bars R, Coassolo P, LeCluyse E. Evaluation of the effect of culture configuration on morphology, survival time, antioxidant status and metabolic capacities of cultured rat hepatocytes. Toxicol. In Vitro 2002; 16(1): 89-99.

46. Martino R, Gilard V, Desmoulin F, Malet-Martino M. Fluorine-19 or phosphorus-31 NMR spectroscopy: a suitable analytical technique for quantitative in vitro metabolic studies of fluorinated or phosphorylated drugs. J. Pharm. Biomed. Anal. 2005; 38(5): 871-891.

47. Yu JX, Kodibagkar VD, Cui W, Mason RP. ${ }^{19} \mathrm{~F}$ : a versatile reporter for non-invasive physiology and pharmacology using magnetic resonance. Curr. Med. Chem. 2005; 12(7): 819-848.

48. Corcoran O, Lindon JC, Hall R, Ismail IM, Nicholson JK. The potential of ${ }^{19} \mathrm{~F}$ NMR spectroscopy for rapid screening of cell cultures for models of mammalian drug metabolism. Analyst, 2001; 126(12): 2103-2106.

49. Bollard ME, Holmes E, Blackledge CA, Lindon JC, Wilson ID, Nicholson JK. ${ }^{1} \mathrm{H}$ and ${ }^{19} \mathrm{~F}$-NMR spectroscopic studies on the metabolism and urinary excretion of mono- and disubstituted phenols in the rat. Xenobiotica, 1996; 26(3): 255-273.

50. Scarfe GB, Tugnait M, Wilson ID, Nicholson JK. Studies on the metabolism of 4-fluoroaniline and 4-fluoroacetanilide in rat: formation of 4-acetamidophenol (paracetamol) and its metabolites via defluorination and N-acetylation. Xenobiotica, 1999; 29(2): 205-216.

51. Williams SN, Callies RM, Brindle KM. Mapping of oxygen tension and cell distribution in a hollow-fiber bioreactor using magnetic resonance imaging. Biotechnol. Bioeng. 1997; 56(1): 56-61.

52. McGovern KA, Schoeniger JS, Wehrle JP, Ng CE, Glickson JD. Gel-entrapment of perfluorocarbons: a fluorine-19 NMR spectroscopic method for monitoring oxygen concentration in cell perfusion systems. Magn. Reson. Med. 1993; 29(2): 196-204.

53. Gross JD, Long RC Jr, Constantinidis I, Sambanis A. Monitoring of dissolved oxygen and cellular bioenergetics within a pancreatic substitute. Biotechnol. Bioeng. 2007; 98(1): 261-270.

54. Goecke-Flora CM. Influence of carbon chain length on the hepatic effects of perfluorinated fatty acids. $\mathrm{A}^{19} \mathrm{~F}$ - and ${ }^{31} \mathrm{P}-\mathrm{NMR}$ investigation. Chem. Res. Toxicol. 1996; 9(4): 689-695.

55. Custer L. Physiological Studies of Hybridoma Cultivation in Hollow Fiber Bioreactors. University of California: Berkeley, CA, 1988.

56. Callies R, Jackson ME, Brindle KM. Measurements of the growth and distribution of mammalian cells in a hollow-fiber bioreactor using nuclear magnetic resonance imaging. Biotechnology (NY), 1994; 12(1): 75-78.

57. Hammer BE, Heath CA, Mirer SD, Belfort G. Quantitative flow measurements in bioreactors by nuclear magnetic resonance imaging. Biotechnology (NY), 1990; 8(4): 327-330.

58. Donoghue C, Brideau M, Newcomer P, Pangrle B, DiBiasio D, Walsh E, Moore $S$. Use of magnetic resonance imaging to analyze the performance of hollow-fiber bioreactors. Ann. NY Acad. Sci. 1992; 665: 285-300.

59. Flendrig LM, la Soe JW, Jorning GG, Steenbeek A, Karlsen OT, Bovee WM, Ladiges NC, te Velde AA, Chamuleau RA. In vitro evaluation of a novel bioreactor based on an integral oxygenator and a spirally wound nonwoven polyester matrix for hepatocyte culture as small aggregates. J. Hepatol. 1997; 26(6): 1379-1392.

60. Macdonald JM, Wolfe SP, Gupta B, Moghe A, Hsu H, Reid LM. Tissue engineering liver in a novel multi-coaxial hollow fiber bioreactor. Free Rad. Biol. Med. 2001; 31: S146.

61. Moghe AK, Gupta BS. Small-diameter blood vessels by weaving: prototyping and modeling. J. Tex. Inst. 2008; 99(5): 467.

62. Van Zijl PC, Moonen CT, Faustino P, Pekar J, Kaplan O, Cohen JS. Complete separation of intracellular and extracellular information in NMR spectra of perfused cells by diffusion-weighted spectroscopy. Proc. Natl. Acad. Sci. USA, 1991; 88(8): 3228-3232.

63. Flint JJ, Lee $C H$, Hansen B, Fey $M$, Schmidig D, Bui JD, King MA, Vestergaard-Poulsen P, Blackband SJ. Magnetic resonance microscopy of mammalian neurons. Neuroimage, 2009; 46(4): 1037-1040.

64. Constantinidis I, Grant SC, Simpson NE, Oca-Cossio JA, Sweeney CA, Mao H, Blackband SJ, Sambanis A. Use of magnetic nanoparticles to monitor alginate-encapsulated betaTC-tet cells. Magn. Reson. Med. 2009; 61(2): 282-290.

65. Michalopoulos GK, Bowen WC, Mule K, Luo J. HGF-, EGF-, and dexamethasone-induced gene expression patterns during formation of tissue in hepatic organoid cultures. Gene Expr. 2003; 11(2): 55-75.

66. Powers MJ, Rodriguez RE, Griffith LG. Cell-substratum adhesion strength as a determinant of hepatocyte aggregate morphology. Biotechnol. Bioeng. 1997; 53(4): 415-426.

67. Capuani G, Miccheli A, Tomassini A, Falasca L, Aureli T, Conti F. Cellular volume determination of alginate-entrapped hepatocytes by MRI diffusion measurements. Artif. Cells Blood Substit. Immobil. Biotechnol. 2000; 28(4): 293-305.

68. Seagle C, Christie MA, Winnike JH, McClelland RE, Ludlow JW, O'Connell TM, Gamcsik MP, Macdonald JM. High-throughput nuclear magnetic resonance metabolomic footprinting for tissue engineering. Tissue Eng. Part C, Methods, 2008; 14(2): 107-118.

69. Planchamp C, Ivancevic MK, Pastor CM, Vallee JP, Pochon S, Terrier F, Mayer JM, Reist M. Hollow fiber bioreactor: new development for the study of contrast agent transport into hepatocytes by magnetic resonance imaging. Biotechnol. Bioeng. 2004; 85(6): 656-665.

70. Roland CR, Naziruddin B, Mohanakumar T, Flye MW. Gadolinium blocks rat Kupffer cell calcium channels: relevance to calciumdependent prostaglandin E2 synthesis and septic mortality. Hepatology, 1999; 29(3): 756-765.

71. Judd RM, Rottman GA, Forder JR, Yin FC, Blackband SJ. Feasibility of ${ }^{19} \mathrm{~F}$ imaging of perfluorochemical emulsions to measure myocardial vascular volume. Magn. Reson. Med. 1992; 28(1): 129-136.

72. Simpson NE, Grant SC, Blackband SJ, Constantinidis I. NMR properties of alginate microbeads. Biomaterials, 2003; 24(27): 4941-4948.

73. Joly A, Desjardins JF, Fremond B, Desille M, Campion JP, Malledant $Y$, Lebreton Y, Semana G, Edwards-Levy F, Levy MC, Clement B. Survival, proliferation, and functions of porcine hepatocytes encapsulated in coated alginate beads: a step toward a reliable bioartificial liver. Transplantation, 1997; 63(6): 795-803.

74. Freyer JP, Fink NH, Schor PL, Coulter JR, Neeman M, Sillerud LO. A system for viably maintaining a stirred suspension of multicellular spheroids during NMR spectroscopy. NMR Biomed. 1990; 3(5): 195-205.

75. O'Leary DJ, Hawkes SP, Wade CG. Indirect monitoring of carbon-13 metabolism with NMR: analysis of perfusate with a closed-loop flow system. Magn. Reson. Med. 1987; 5(6): 572-577.

76. De Bartolo L, Jarosch-Von Schweder G, Haverich A, Bader A. A novel full-scale flat membrane bioreactor utilizing porcine hepatocytes: cell viability and tissue-specific functions. Biotechnol. Prog. 2000; 16(1): 102-108.

77. McClelland RE, Coger RN. Effects of enhanced $\mathrm{O}(2)$ transport on hepatocytes packed within a bioartificial liver device. Tissue Eng. 2004; 10(1-2): 253-266.

78. Kusumi T, Ishihara K, Mizumoto H, Nakazawa K, ljima H, Funatsu K, Kajiwara T. Evaluation of a bioreactor with stacked sheet shaped organoids of primary hepatocytes. J. Biosci. Bioeng. 2009; 107(5): 552-555.

79. McClelland RE, Coger RN. Use of micropathways to improve oxygen transport in a hepatic system. J. Biomech. Eng. 2000; 122(3): 268-273. 
80. Allen JW, Bhatia SN. Formation of steady-state oxygen gradients in vitro: application to liver zonation. Biotechnol. Bioeng. 2003; 82(3): 253-262.

81. Catapano G, Patzer JF 2nd, Gerlach JC. Transport advances in disposable bioreactors for liver tissue engineering. Adv. Biochem. Eng. Biotechnol. 2010; 115: 117-143.

82. Powers MJ, Griffith-Cima L. Motility behavior of hepatocytes on extracellular matrix substrata during aggregation. Biotechnol. Bioeng. 1996; 50(4): 392-403.

83. McLaughlin BE, Tosone CM, Custer LM, Mullon C. Overview of extracorporeal liver support systems and clinical results. Ann. NY Acad. Sci. 1999; 875: 310-325.

84. Ring A, Gerlach J, Peters G, Pazin BJ, Minervini CF, Turner ME, Thompson RL, Triolo F, Gridelli B, Miki T. Hepatic maturation of human fetal hepatocytes in four-compartment three-dimensional perfusion culture. Tissue Eng. Part C: Methods, 2010; 16(5): 835-845.

85. Cohen SM, Ogawa S, Shulman RG. ${ }^{13} \mathrm{C}$ NMR studies of gluconeogenesis in rat liver cells: utilization of labeled glycerol by cells from euthyroid and hyperthyroid rats. Proc. Natl. Acad. Sci. USA, 1979; 76(4): 1603-1609.
86. Cohen SM, Shulman RG. ${ }^{13} \mathrm{C}$ n.m.r. studies of gluconeogenesis in rat liver suspensions and perfused mouse livers. Philos. Trans. R. Soc. London B: Biol. Sci. 1980; 289(1037): 407-411.

87. Cohen SM, Rognstad R, Shulman RG, Katz J. A comparison of ${ }^{13} \mathrm{C}$ nuclear magnetic resonance and ${ }^{14} \mathrm{C}$ tracer studies of hepatic metabolism. J. Biol. Chem. 1981; 256(7): 3428-3432.

88. Cohen SM, Glynn P, Shulman RG. ${ }^{13} \mathrm{C}$ NMR study of gluconeogenesis from labeled alanine in hepatocytes from euthyroid and hyperthyroid rats. Proc. Natl. Acad. Sci. USA, 1981; 78(1): 60-64.

89. Brooks WM, Moxon LN, Field J, Irving MG, Doddrell DM. In vitro metabolism of $\left[2-{ }^{13} \mathrm{C}\right]$-ethanol by ${ }^{1} \mathrm{H}$ NMR spectroscopy using ${ }^{13} \mathrm{C}$ decoupling with the reverse dept polarization-transfer pulse sequence. Biochem. Biophys. Res. Commun. 1985; 128(1): 107-112.

90. Goffe RAA, Genespan Corporation (Redmond, WA, USA), assignee. High performance cell culture bioreactor and method. United States Patent 5622857, 1997.

91. Ji H, Graczyk-Milbrandt G, Osbakken MD, Friedman MI. Interactions of dietary fat and 2,5-anhydro-D-mannitol on energy metabolism in isolated rat hepatocytes. Am. J. Physiol. Regul. Integr. Comp. Physiol. 2002; 282(3): R715-R720. 